\title{
A New Method for Characterizing Very Low-Mass Companions with Low-Resolution Near-Infrared Spectroscopy
}

\author{
Emily L. Rice, ${ }^{1,2}$ Rebecca OpPenheimer, ${ }^{2}$ Neil Zimmerman, ${ }^{3}$ Lewis C. Roberts JR., ${ }^{4}$ And Sasha Hinkley ${ }^{5,6}$ \\ Received 2014 July 22; accepted 2015 March 26; published 2015 April 24
}

\begin{abstract}
We present a new and computationally efficient method for characterizing very low-mass companions using low-resolution $(R \sim 30)$, near-infrared $(Y J H)$ spectra from high-contrast imaging campaigns with integral field spectrograph (IFS) units. We conduct a detailed quantitative comparison of the efficacy of this method through tests on simulated data comparable in spectral coverage and resolution to the currently operating directimaging systems around the world. In particular, we simulate Project 1640 data as an example of the use, accuracy, and precision of this technique. We present results from comparing simulated spectra of M, L, and T dwarfs with a large and finely sampled grid of synthetic spectra using Markov-chain Monte Carlo techniques. We determine the precision and accuracy of effective temperature and surface gravity inferred from fits to PHOENIX dusty and cond, which we find reproduce the low-resolution spectra of all objects within the adopted flux uncertainties. Uncertainties in effective temperature decrease from $\pm 100-500 \mathrm{~K}$ for $\mathrm{M}$ dwarfs to as small as $\pm 30 \mathrm{~K}$ for some $\mathrm{L}$ and $\mathrm{T}$ spectral types. Surface gravity is constrained to within $0.2-0.4$ dex for mid-L through T dwarfs, but uncertainties are as large as 1.0 dex or more for $\mathrm{M}$ dwarfs. Results for effective temperature from low-resolution $Y J H$ spectra generally match predictions from published spectral type-temperature relationships except for L-T transition objects and young objects. Single-band spectra (i.e., narrower wavelength coverage) result in larger uncertainties and often discrepant results, suggesting that high-contrast IFS observing campaigns can compensate for low spectral resolution by expanding the wavelength coverage for reliable characterization of detected companions. We find that $\mathrm{S} / \mathrm{N} \sim 10$ is sufficient to characterize temperature and gravity as well as possible given the model grid. Most relevant for direct-imaging campaigns targeting young primary stars is our finding that low-resolution near-infrared spectra of known young objects, compared to field objects of the same spectral type, result in similar best-fit surface gravities but lower effective temperatures, highlighting the need for better observational and theoretical understanding of the entangled effects of temperature, gravity, and dust on near-infrared spectra in cool low-gravity atmospheres.
\end{abstract}

Online material: color figures.

\section{INTRODUCTION}

Extrasolar planets have been indirectly detected for over 20 years, and there are more nearly 2000 known systems, with over twice as many Kepler candidates awaiting confirmation. ${ }^{7}$ Indirect detection techniques can provide mass, radius, and bulk composition measurements for extrasolar planets, but they are

\footnotetext{
${ }^{1}$ Department of Engineering Science and Physics, College of Staten Island, City University of New York, Staten Island, NY 10314; emily.rice@csi.cuny .edu.

${ }^{2}$ Department of Astrophysics, American Museum of Natural History, New York, NY 10024.

${ }^{3}$ Department of Mechanical and Aerospace Engineering, Princeton University, Princeton, NJ 08544.

${ }^{4}$ Jet Propulsion Laboratory, California Institute of Technology, Pasadena, CA 91109.

${ }^{5}$ Astronomy Department, California Institute of Technology, Pasadena, CA 91125.

${ }^{6}$ Department of Physics and Astronomy, University of Exeter, Devon EX4 4QL, UK.

${ }^{7}$ http://exoplanetarchive.ipac.caltech.edu/
}

limited in their utility to derive atmospheric properties, which are essential to testing formation scenarios (e.g., Fortney et al. 2008; Spiegel \& Burrows 2012). Recent direct imaging of extrasolar planets (e.g., Kalas et al. 2008; Marois et al. 2008, 2010; Lagrange et al. 2010; Currie et al. 2014) and studies of transiting planets in secondary eclipse (e.g., Deming et al. 2007; Knutson et al. 2008; Sing \& López-Morales 2009; Fortney et al. 2013) have expanded the field to include pseudospectral studies via photometric observations made at multiple wavelengths, including narrowband filters (Janson et al. 2010).

Integral field spectroscopy will rapidly expand the study of substellar companion and exoplanetary atmospheres. In the last few years, direct detection and spectroscopy has revealed a diversity of atmospheric properties, even within the same planetary system (Patience et al. 2010; Bowler et al. 2010; Barman et al. 2011a; Konopacky et al. 2013; Oppenheimer et al. 2013; Chilcote et al. 2015). Several ground-based high-contrast imaging instruments have been specifically designed to directly image extrasolar planets, including the Near-Infrared Coronagraphic Imager (NICI) on Gemini-South (Chun et al. 2008), the High Contrast 
Instrument for the Subaru Next Generation Adaptive Optics (HiCIAO) and later the Coronagraphic High Angular Resolution Imaging Spectrograph on (CHARIS) on Subaru (Suzuki et al. 2010; McElwain et al. 2012), Project 1640 (P1640) on the Palomar Observatory Hale 200 inch telescope (Hinkley et al. 2011b; Oppenheimer et al. 2012), the Gemini Planet Imager (GPI) on Gemini-South (Macintosh et al. 2014), and the SpectroPolarimetric High-contrast Exoplanet Research (SPHERE) on the VLT (Beuzit et al. 2008).

P1640, GPI, and SPHERE include integral field spectrograph (IFS) units that produce low-resolution spectra (spectral sampling $R=[\lambda / \Delta \lambda] \sim 30-60)$ of directly imaged companions in the near-infrared $(\sim 1-2 \mu \mathrm{m})$. The high-contrast ratios achieved by these instruments greatly expands the parameter space of the companions for which spectroscopy can be obtained, including M, L, and T dwarf and planetary companions around BAFG stars (Zimmerman et al. 2010; Hinkley et al. 2010, 2011a; Roberts et al. 2012; Hinkley et al. 2013; Chilcote et al. 2015; Crepp et al. 2015) and simultaneous observations of multiple-planet systems (Oppenheimer et al. 2013), all at Solar System-like separations (4-40 AU). The low-resolution of spectra obtained by these instruments are crucial for confirming the nature of the companions and for constraining their atmospheric properties and formation scenarios.

The primary purpose of IFS units on high-contrast instruments is to distinguish faint companions from residual light from the primary star that has been diffracted through the instrument in a complex pattern (Oppenheimer \& Hinkley 2009). The IFS exploits the wavelength dependence of the diffraction in order to separate speckles from faint objects, which will remain at the same position on the image plane while the speckles change position with wavelength (Sparks \& Ford 2002). The spectral sampling and coverage of IFS units is motivated by speckle suppression rather than spectral characterization of detected companions, and is limited by the engineering complexities of the lenslet arrays and the pixel and chip size of infrared detectors.

Very low-mass stars, brown dwarfs, and planetary-mass companions have the potential to be efficiently characterized by comparing observed low-resolution spectra to synthetic spectra from model atmospheres, as long as the selected wavelength regime contains significantly broad spectral features that are sensitive to atmospheric properties like effective temperature, surface gravity, and metallicity. Spectral fitting thus avoids assumptions about age, radius, and mass, as well as the evolutionary models that relate these quantities, e.g., Burrows et al. (1997); Chabrier et al. (2000); Baraffe et al. (2002); Fortney et al. (2007). As higher contrast and lower mass objects are imaged and observed spectroscopically, the ability to conduct such comparisons efficiently and reliably is needed.

We have developed a method to derive atmospheric parameters and uncertainties by fully mapping a broad temperaturegravity parameter space using thousands of synthetic spectra and linearly interpolating between calculated models. We com- pare simulated P1640 spectra (described in $\S 2$ ) of field M, L, and $\mathrm{T}$ dwarfs and young $\mathrm{M}$ and $\mathrm{L}$ spectral-type objects to synthetic spectra calculated with the PHOENIX model atmosphere code (§ 3) using a Markov-chain Monte Carlo (MCMC)-based fitting procedure described in $\S 4$. $\S 5$ presents the resulting probability distribution functions and best-fit model spectra, describes the constraints on parameters of effective temperature and surface gravity, compares results obtained using different subsets of the simulated spectra, and discusses the dependence of best-fit parameters and uncertainties on the noise level in the simulated spectra. The conclusions of this work are presented in $\S 6$.

\section{SPECTRAL TEMPLATES AND SIMULATED SPECTRA}

In order to test our fitting procedure over a range of effective temperatures (hence companion masses), we constructed lowresolution $(\Delta \lambda \sim 300 \AA)$ spectra from moderate-resolution, high signal-to-noise ratio $(\mathrm{S} / \mathrm{N})$ observed spectra of field $\mathrm{M}$, $\mathrm{L}$, and $\mathrm{T}$ dwarfs and young $\mathrm{M}$ and $\mathrm{L}$ dwarfs. The original observational spectra are henceforth referred to as "template" spectra and the constructed lower resolution spectra as "simulated" spectra. The creation of simulated low-resolution nearinfrared spectra from the template spectra is described below.

\subsection{Spectral Templates}

The sample of objects used as spectral templates is summarized in Table 1. Their spectral types range from M1 to T4.5, spanning the range of effective temperatures expected for lowmass companions that can be detected by the current generation of high-contrast integral field spectrographs (Beichman et al. 2010). All template spectra were obtained with the SpeX spectrometer on the NASA Infrared Telescope Facility (IRTF; Rayner et al. 2003) in cross-dispersed mode, covering $0.81-2.4 \mu \mathrm{m}$ with a resolving power $(R=\lambda / \Delta \lambda)$ of at least 2000, with the exception of the spectrum of PSO J318.5338-22.8603, which was obtained on the GNIRS spectrograph (Elias et al. 2006) on the Gemini-North $8.1 \mathrm{~m}$ Telescope in cross-dispersed mode, covering $0.95-2.5 \mu \mathrm{m}$ with $R \sim 1700$.

High-contrast direct-imaging surveys will primarily target young stars for which low-mass companions are likely to be still contracting, thus they have lower surface gravity than field-age objects of the same mass. Template objects include both field (ages $>1$ Gyr) M, L, and T dwarfs and young ( 10-100 Myr) M and L dwarfs. Spectra of field objects are from the IRTF Spectral Library (Cushing et al. 2005; Rayner et al. 2009). The young objects are an M8.5 $\gamma$ (Rice et al. 2010c), an L0 $\gamma$ (Kirkpatrick et al. 2006), an L5 $\gamma$ (Faherty et al. [2013]; see Cruz et al. [2009] for complete description of the $\gamma$ gravity suffix), and an L7 VL-G (Allers \& Liu 2013; Liu et al. 2013). These young objects are high-probability members of $\beta$ Pictoris, Tucana-Horologium, AB Doradus, and $\beta$ Pictoris, respectively (Rice et al. 2010c; Gagné et al. 2014; Faherty et al. 2013; 
TABLE 1

IRTF/SPEX SPECTRA

\begin{tabular}{lcccc}
\hline \hline \multicolumn{1}{c}{ Spectral type } & Object name & R.A. (h, m, s) & Decl. $\left({ }^{\circ},,^{\prime}, "\right)$ & Ref. \\
\hline Field templates & & & & \\
\hline M1 & HD 42581 & 061034.6 & -215153 & 1 \\
M5 & Gl 866ABC & 223833.7 & -151757 & 1 \\
M8 & Gl 752B & 191655.3 & +051011 & 1,2 \\
L1 & 2MASS J1439+1929 & 143928.4 & +192915 & 1 \\
L5 & 2MASS J1507-1627 & 150747.7 & -162739 & 1 \\
L8 & DENIS J0255-4700 & 025503.6 & -470051 & 1 \\
T2 & SDSS J1254-0122 & 125453.9 & -012247 & 1 \\
T4.5 & 2MASS J0559-1404 & 055919.1 & -140449 & 1 \\
\hline Young templates & & & & \\
\hline M8.5 $\gamma$ & 2MASS J0608-2753 & 060852.8 & -275358 & 3 \\
L0 $\gamma$ 2MASS J0141-4633 & 014158.23 & -463357.4 & 4 \\
L5 $\gamma$ L3 VL-G & 2MASS J0355+1133 & 035523.4 & +113344 & 5,6 \\
L7 \pm 1(IR) VL-G & PSO J318.5338-22.8603 & 211408.026 & -225135.84 & 7 \\
\hline
\end{tabular}

NotE.-References. - (1) Rayner et al. 2009; (2) Cushing et al. 2005; (3) Rice et al. 2010c; (4) Kirkpatrick et al. 2006; (5) Faherty et al. 2013; (6) Allers \& Liu 2013; (7) Liu et al. 2013.

a Although Gl 866ABC is a triple system, Rojas-Ayala et al. (2012) find it to be close to Solar metallicity $([\mathrm{M} / \mathrm{H}]=0.05 \pm 0.12)$ with a temperature similar to single M5 dwarfs according to analysis of an unresolved $R \sim 2700 \mathrm{~K}$-band spectrum.

Liu et al. 2013), and are among the closest photometric and spectroscopic analogs of directly imaged gas giant planets for which detailed analysis is possible (see, e.g., Faherty et al. 2013; Allers \& Liu 2013).

\subsection{Simulated High-Contrast IFS Spectra}

We simulate low-resolution near-infrared spectra of very low-mass stellar and substellar companions that can be obtained by high-contrast IFS units in order to test the accuracy and precision of physical parameters inferred from model fits to these spectra. We report results for spectra representative of the resolution and wavelength range for Project 1640, which has a single observing mode covering the $Y J H$ band with a sampling of $\Delta \lambda \sim 300 \AA$.

Project 1640 has operated since 2008 on the Hale telescope in two distinct phases, Phase 1 and Phase 2, separated by a substantial instrument upgrade (new detector, filters, and increased sensitivity; Oppenheimer et al. [2012]). Phase 1 (July 2008 to June 2012) produced $J H(1.1-1.8 \mu \mathrm{m})$ spectra at $300 \AA_{\text { pixel }}^{-1}$ resolution with one instrument setting (see Hinkley et al. 2008, 2011b). Phase 2 upgrades (begun June 2012) extended the wavelength coverage to $Y J H(0.9-1.8 \mu \mathrm{m})$ and slightly increased the spectral sampling to $286 \AA$ pixel $^{-1}$ (Oppenheimer et al. 2012, 2013). Simulated P1640 spectra were created from SpeX/IRTF template spectra for both phases, but the results of this paper focus on fits to Phase 2 spectra.

The first step in the creation of simulated P1640 spectra is to define the wavelength channels based on the data reduction and spectral extraction procedures developed by the instrument team (Zimmerman et al. 2011; Oppenheimer et al. 2013). At each wavelength channel, the flux density $\left(F_{\lambda}\right)$ value of the simulated spectrum was set to the median of the flux density from the template spectrum within the wavelength range of the channel. The quoted resolution of the binned spectra represents the spectral sampling rather than the full-width at halfmaximum of the instrumental profile. The simulated spectra were then normalized to the maximum value in the P1640 wavelength range. The template IRTF spectra and simulated P1640 spectra are plotted in Figures 1 and 2.

The simulated spectra are significantly higher $\mathrm{S} / \mathrm{N}$ than the typical spectra of very low-mass companions obtained with ground-based high-contrast integral field spectrographs (e.g., Oppenheimer et al. 2013; Chilcote et al. 2015). Therefore, for the primary analysis described in $\S 4$, the uncertainty on the simulated spectra was set to $\mathrm{S} / \mathrm{N}=10$ at the peak flux value and assigned a constant noise at each flux point, resulting in lower $\mathrm{S} / \mathrm{N}$ for lower flux values. The actual uncertainties on these flux points (i.e., errors on the template spectra flux added in quadrature within each wavelength channel) the are much smaller; therefore, we test the dependence of best-fit parameters and uncertainties on $\mathrm{S} / \mathrm{N}$ via several "resampling" tests described in $\S 5.7$.

The simulated spectra do not explicitly include any instrumental systematics such as speckles or cross-talk (see Oppenheimer \& Hinkley 2009). The effects of residual uncorrected wavefront aberrations, or speckles, coinciding with a detected companion in the extracted image cube is prohibitively time-consuming to predict and incorporate into the our analysis, although they are minimized with current data reduction and spectral extraction procedures (Pueyo et al. 2012; Oppenheimer et al. 2013; Fergus et al. 2014). Another potentially significant instrumental effect 

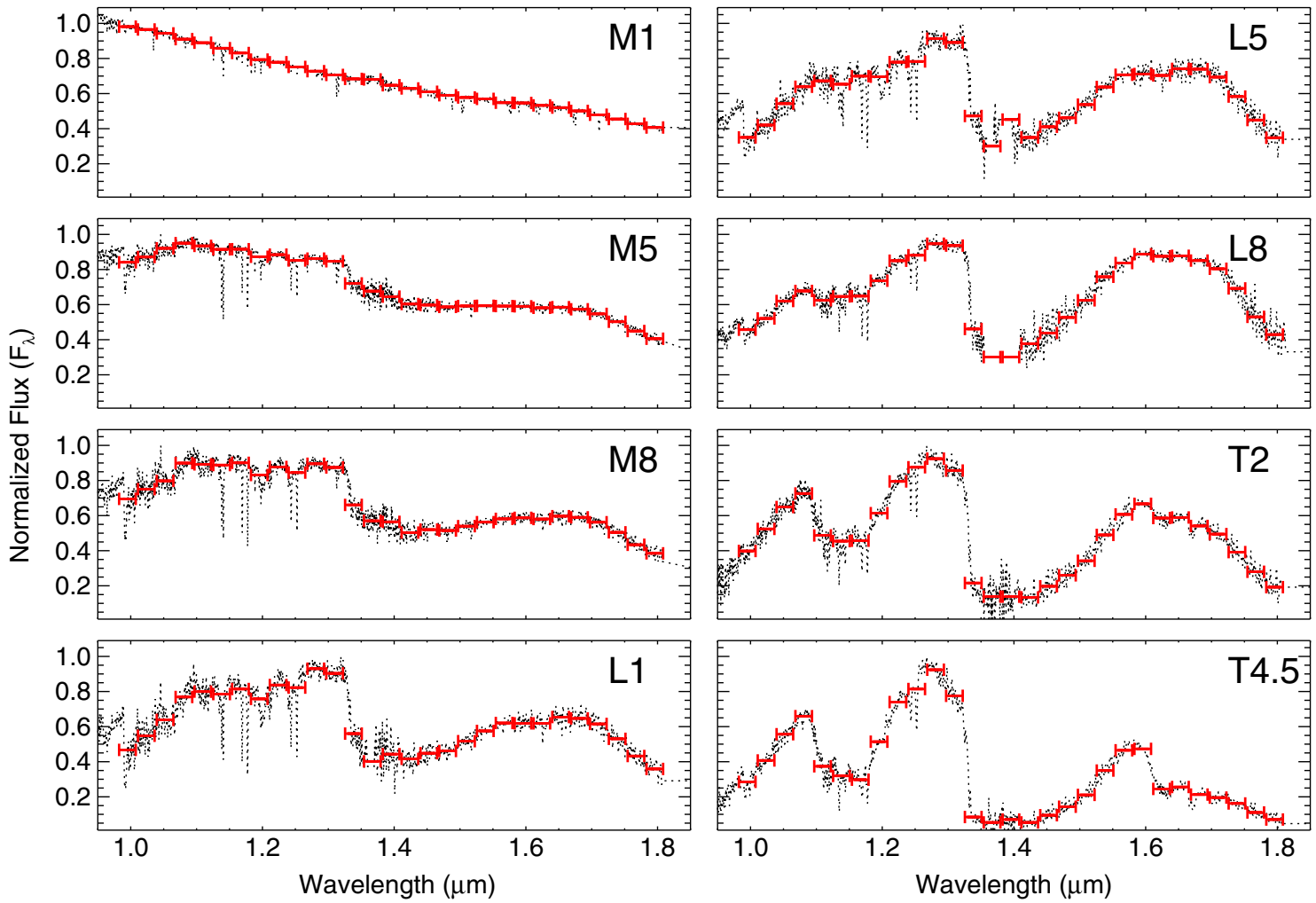

FIG. 1.-Observed (dashed lines) and simulated (red bars) near-infrared spectra of field M, L, and T dwarfs from IRTF/SpeX library spectra (Cushing et al. 2005; Rayner et al. 2009). The binned spectra simulate the wavelength coverage and spectral sampling of P1640 YJH spectra (Phase 2). The calculation of the simulated spectra is described in $\S 2.2$. The spectral template sources are listed in Table 1. See the online edition of the PASP for a color version of this figure.

is cross-talk, or light from different wavelengths leaking into neighboring spectra on the detector. For Phase 2 data, cross-talk has been reduced to a maximum of $1.5 \%$ and average of $0.4 \%$ across all wavelengths with better focusing of the instrument, an improved blocking filter, and optimized extraction methods (Oppenheimer et al. 2013).

\section{MODEL ATMOSPHERES AND SYNTHETIC SPECTRA}

Model atmospheres and synthetic spectra were calculated using the PHOENIX atmosphere code (Hauschildt et al. 1997, 1999). For an effective temperature range $1400-4500 \mathrm{~K}$, appropriate for $\mathrm{M}$ and $\mathrm{L}$ dwarfs, the dusty version of the PHOENIX code was used, as presented in Rice et al. (2010a), Schlieder et al. (2012), and Roberts et al. (2012). The cond version of the PHOENIX code was used for an effective temperature range of 950-2000 K, appropriate for mid- to late- L dwarfs and earlyto mid-T dwarfs as presented in Rice et al. (2010b) and similar to del Burgo et al. (2009).

The dusty and cond versions of the PHOENIX models represent limiting cases of dust treatments. In the dusty version, dust is created in thermochemical equilibrium, assumed to be made of spherical single-species grains with a power-law size distribution, and remains static in the atmosphere as a source of opacity. In the cond version, dust is created identically but the opacity is not considered in calculation of the emergent spectrum, thus mimicking perfectly efficient removal of the dust to layers below the photosphere. These limiting cases have been shown to work well for objects hotter than mid-M type (e.g., Rice et al. 2010a) and cooler than the L-T transition (e.g., del Burgo et al. 2009; Rice et al. 2010b). While more sophisticated dust treatments have been implemented in other atmosphere models, we use PHOENIX models because we have access to a complete and finely sampled model grid over the required range of effective temperature and surface gravity.

To test the veracity of our PHOENIX dusty and cond synthetic spectra compared to low-resolution near-infrared spectra of $\mathrm{M}, \mathrm{L}$, and $\mathrm{T}$ dwarfs, we added a nuisance parameter to the fitting procedure described in $\S 4$ below. This parameter is added in quadrature to the actual uncertainty on the binned flux points (i.e., not the adopted uncertainty for $\mathrm{S} / \mathrm{N} \leq 10$ ) and allowed to vary. The $T_{\text {eff }}$ and $\log (g)$ results from these fits marginalized over the tolerance parameter were within the $1-\sigma$ uncertainties of the reported results for all spectra and the nuisance parameter was always less than 0.10 , with typical peaks around 5\%. This indicates that the systematic differences between the models and the simulated spectra are $\sim 5 \%$ and as little as $1-2 \%$ for the field $\mathrm{M}$ dwarfs. 

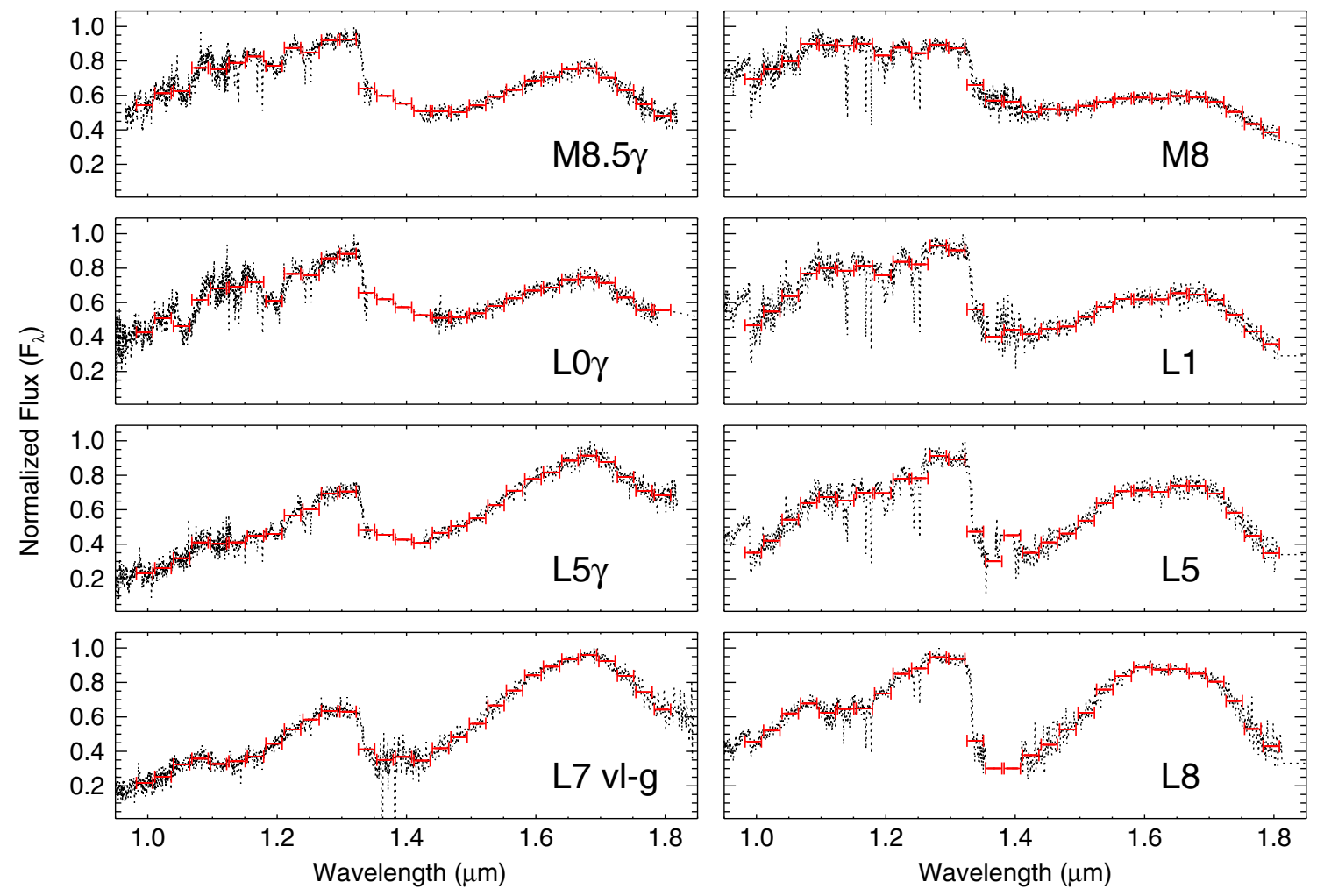

FIG. 2.-Left: Observed (dashed lines) and simulated (red bars) near-infrared spectra of young M and L spectral type objects (Rice et al. 2010c; Kirkpatrick et al. 2006; Faherty et al. 2013; Allers \& Liu 2013; Liu et al. 2013). Right: Their closest field-age counterparts in our sample. The binned spectra simulate the wavelength coverage and spectral sampling of P1640 Y JH spectra. The calculation of the simulated spectra is described in $\S 2.2$. The spectral template sources are listed in Table 1. See the online edition of the PASP for a color version of this figure.

For the model grids, atmospheric temperature-pressure structures were calculated in intervals of $50 \mathrm{~K}$ and with surface gravities ranging from $\log (g)=3.0-6.0$ [cgs] in 0.1 dex intervals, resulting in 1952 dusty models and 682 cond models. We include surface gravities up to $\log (g)=6.0$ (some unphysical) in our model grid in order to test the inherent sensitivity of the spectra to surface gravity in the absence of independent age constraints and to avoid a possibly artificial upper limit at $\log (g)=5.5$. This also enables more reliable uncertainty estimates at high surface gravities because the probability distributions are not restricted to the physically allowed values. All atmosphere structures were calculated at solar metallicity.

The calculation of a converged atmospheric structure with the PHOENIX code also produces a synthetic spectrum at $4 \AA$ sampling from $10 \AA$ to $5 \mu \mathrm{m}$; therefore, the models oversample the simulated P1640 spectra by a factor of $\sim 70$. Synthetic spectra matching the wavelength range and spectral sampling of P1640 are created following the same method used for simulated spectra, described in $\S 2.2$ above. Binned synthetic spectra for the range of effective temperatures and surface gravities used in the fitting procedure are shown in Figure 3. Within the model fitting procedure (described in $\S 4$ ) the calculated synthetic spectra are first linearly interpolated in flux to produce spectra with the desired temperature and gravity values, then binned to match the wavelength coverage and spectral sampling of the simulated P1640 spectra as described in § 2.2.

\section{SPECTRAL FITTING METHOD}

We use a two-step spectral fitting procedure to derive best-fit physical parameters and uncertainties. The fitting procedure uses a grid of model spectra that are $\sim 10$ times higher spectral sampling than the comparison (i.e., observed or simulated) spectrum and calculated at fine enough sampling in parameter space to allow reliable linear interpolation in flux (as described in Rice et al. [2010a] and below). The linear interpolation is done on the fly by the fitting code, effectively mimicking a continuous grid of model spectra in the specified parameter space. In the current analysis, we consider model parameters of effective temperature and surface gravity. Future work will include super-solar abundances expected for extrasolar planets created via core accretion (e.g., Fortney et al. 2008; Öberg et al. 2011). The fitting procedure is derived from the methods of Rice et al. (2010a) and updated in Roberts et al. (2012), Hinkley et al. (2013), and Crepp et al. (2015).

The first step of the procedure uses the calculated model grids described in $\S 3$. For each synthetic spectrum we calculate 


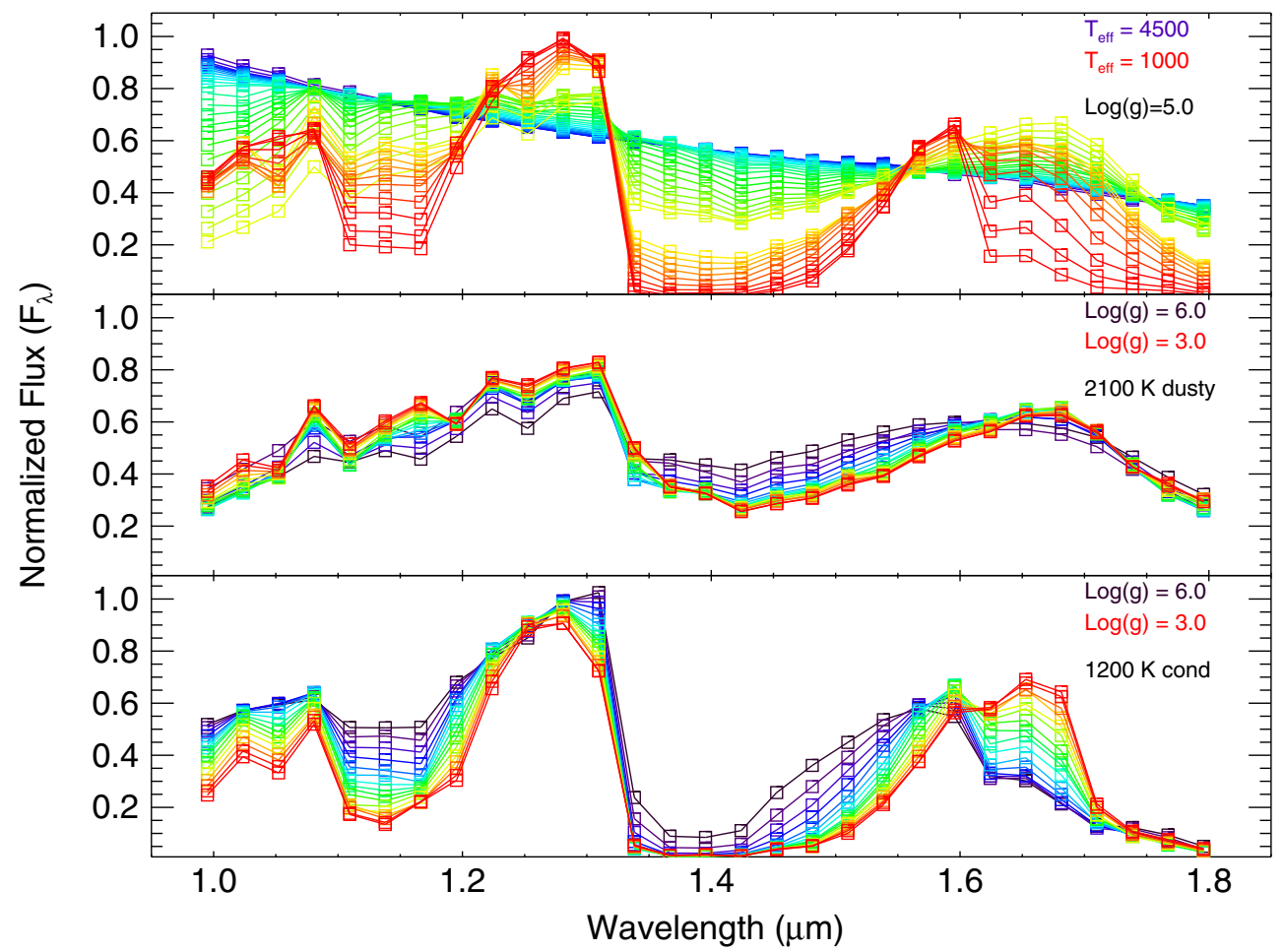

FIG. 3. - Synthetic spectra calculated with PHOENIX model atmosphere code trimmed and binned to match P1640 spectra as described in § 3. Top: Spectra for the complete range of model temperatures in $100 \mathrm{~K}$ increments at a fixed surface gravity $\log (g)=5.0$ [cgs] expected for field objects. The dusty models are shown for a fixed $T_{\text {eff }} \geq 2000 \mathrm{~K}$, and cond models are shown for $T_{\text {eff }} \leq 1900 \mathrm{~K}$, hence the gap between spectra apparent at $\sim 1.4 \mu \mathrm{m}$. Middle: Spectra for the range of surface gravities at $2100 \mathrm{~K}$ (approximately late-M/early-L spectral types) from dusty models. Bottom: The same at a fixed $T_{\text {eff }}=1200 \mathrm{~K}$ (approximately early-mid T dwarfs) from cond models. See the online edition of the PASP for a color version of this figure.

a goodness-of-fit value based on the weighted sum of squared errors (see Cushing et al. 2008). This calculation provides a coarse overview of the entire parameter range. Example results of this step are presented in Figure 4 for the $Y J H$ simulated spectra of the field M5, L1, and T4.5 objects.

The second step of the procedure uses a MetropolisHastings-based MCMC method to derive the posterior probability distribution over the model parameters. We assume an uninformative prior, i.e., one that is uniform over the range of parameters in the model grid. The uniform prior ignores prior knowledge about the objects that would make certain parameter regions higher or lower probability. We use it in order to present the most broadly applicable results and uncertainties without incorporating potentially inaccurate prior assumptions. The chain is initialized using the parameters of the model with the lowest (best) goodness-of-fit values from the previous step. Subsequent models are generated on the fly via linear interpolation of flux points from the precalculated grid in order to analyze an effectively continuous grid of models. We tested the veracity of linear interpolation in temperature by comparing spectra created via linear interpolation between two models $100 \mathrm{~K}$ apart to the calculated model with the same temperature. The mean difference for each flux point was $0.7 \%$ for the dusty models and $1.4 \%$ for the cond models, each with $\sigma \sim 2 \%$, so we expect the systematic differences from linear interpolation to be at most $1-2 \%$.

The uncertainties on the simulated or observed spectra are used to calculate the $\chi^{2}$ for the samples drawn from the posterior distribution. The "jump size" (width of the normal distribution from which the new model parameters are randomly selected) was adjusted for each object to produce an acceptance rate (number of accepted jumps divided by the total number of links) of $\sim 0.3-0.4$. The jump sizes varied from 12.5 to $1450 \mathrm{~K}$ in effective temperature and 0.025 to 2.9 dex in surface gravity, with larger jump sizes for earlier spectral types. If a jump took the parameter outside the calculated model grid, then the interpolation step sets the parameter to just inside the grid.

The MCMC chain length for each spectrum is $10^{6}$ links, where each link is a sample from the posterior distribution, whether or not the new sample is accepted into the chain. Typically in MCMC analysis the first $\sim 10 \%$ of the links are considered "burn-in" and not included in the final analysis; however, initializing the chain with the parameters with the lowest global goodness of fit values effectively removes the need for a burn in period. We confirm this by comparing the mean and standard deviations of the first $10^{5}$ steps in the chain with the $9 \times 10^{5}$ 

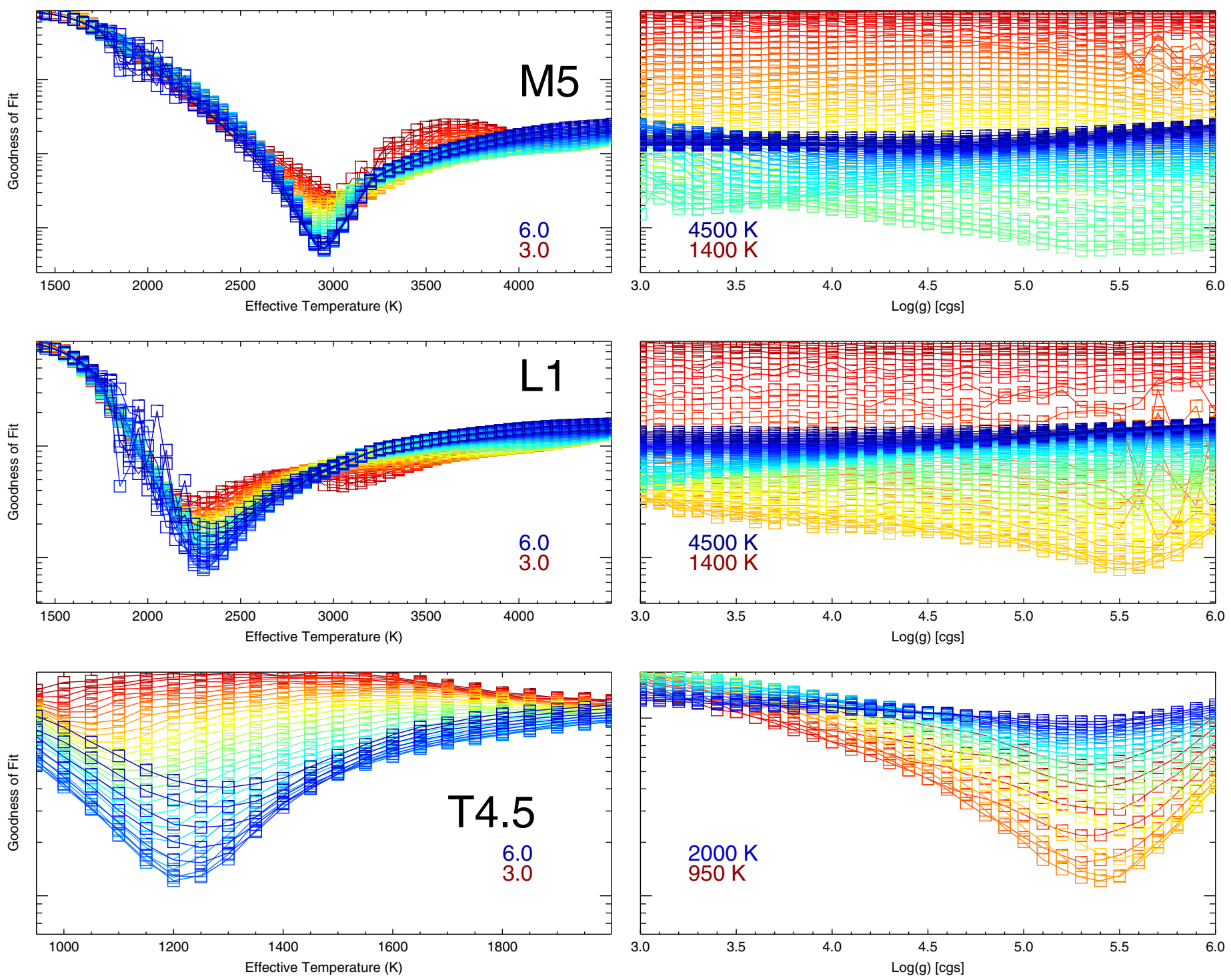

FIG. 4.-Goodness-of-fit values for model spectra as a function of effective temperature (left) and surface gravity (right) for the simulated P1640 YJH spectra from the M5 (top), L1 (middle), and T4.5 (bottom) spectral templates. This represents the first step in the fitting process and provides an overview of the complete parameter space. In these examples, the lowest goodness-of-fit values are for model parameters $2950 \mathrm{~K}$ and $\log (g)=5.3$ [cgs] for the M5 template, $2300 \mathrm{~K}$ and $\log (g)=5.5$ [cgs] for the $\mathrm{L} 1$ and $1200 \mathrm{~K}$, and $\log (g)=5.3$ [cgs] for the T4.5. These values are used as starting points for the MCMC procedure. The overview of parameter space provided by this step in the fitting procedure demonstrates that the low-resolution near-infrared spectra of very low-mass objects are sensitive to temperature (as expected) and are increasingly sensitive to surface gravity for later spectral types. See the online edition of the PASP for a color version of this figure.

postburn links, which were nearly identical. Therefore, the complete $10^{6}$ link chains are used in analysis presented in $\S 5$.

For preliminary tests of the fitting procedure, spectra were fit with the both dusty and the cond models with the appropriate temperature ranges for each dust treatment (described in $\S 3$ ). This, as expected, resulted in poor fits to the M dwarfs using the cond models and to the T dwarfs using the dusty models. For the L dwarfs, the dusty model fits resulted in qualitatively better fits to the data; therefore, only the dusty models were used for the complete analysis of the $\mathrm{L}$ dwarfs. Preliminary tests were also run with fewer links $\left(10^{4}-10^{5}\right)$ with similar best-fit results, indicating that the $10^{6}$ link chains are converged.

P1640 spectra include the $\mathrm{H}_{2} \mathrm{O}$ absorption band at $\sim 1.4 \mu \mathrm{m}$ that is present in objects $\sim \mathrm{M} 4$ and later as well as in the Earth's atmosphere. Because the signal is low and the correction for telluric absorption is often poor, this region is typically excluded from analysis for observed spectra (e.g., Roberts et al. 2012; Oppenheimer et al. 2013). Therefore, we also fit "trimmed" simulated P1640 spectra by excluding four flux points in the $\sim 1.4 \mu \mathrm{m} \mathrm{H}_{2} \mathrm{O}$ absorption band. GPI and SPHERE cover the $Y J H K$ spectral range with several individual filters (e.g., $Y$, 


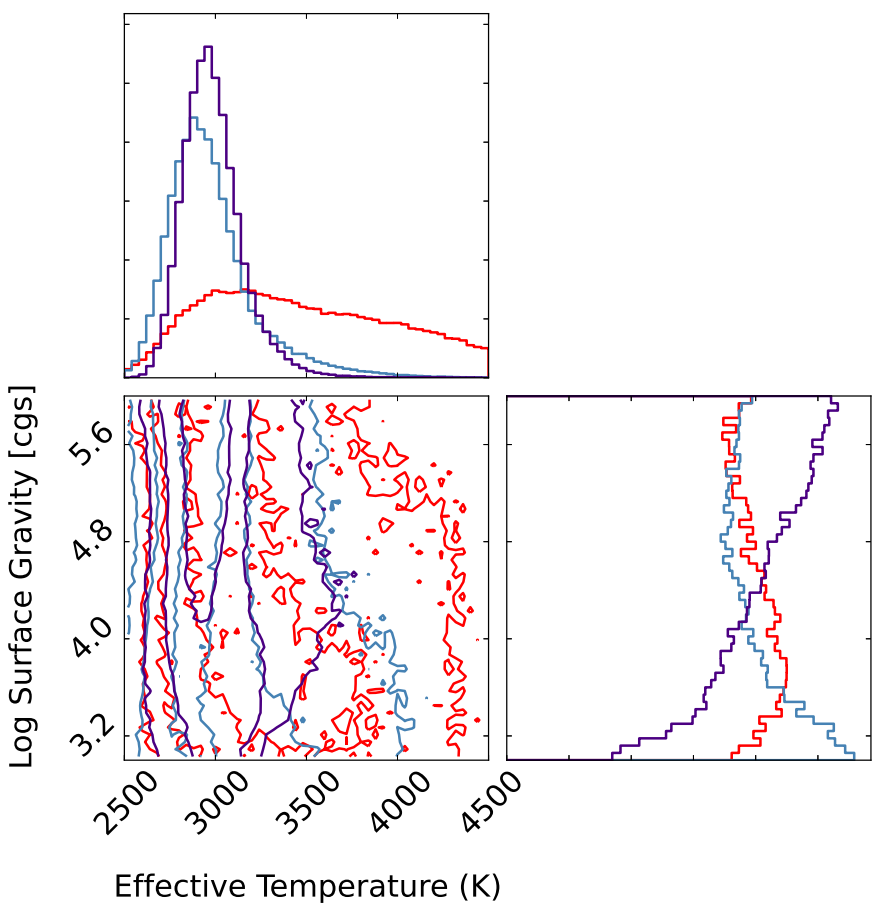

FIG. 5.-MCMC results for the field M5 spectral template object for: complete $Y J H$ spectrum (purple), $Y J$ spectrum (blue), and $H$ spectrum (red). Bottom left: $1-\sigma, 2-\sigma$, and $3-\sigma$ contours of the posterior distribution functions for both parameters. Top, bottom right: Histograms for temperature and surface gravity, respectively, marginalized over the other parameter. The distributions for the $Y J H$ and $Y J$ spectra are much narrower in temperature than in surface gravity. The $Y J H$ - and $Y J$-band spectra produce similar temperature results, but the $H$-band distribution has an only slightly sloping tail toward higher temperatures. See the online edition of the PASP for a color version of this figure.

$J, H, K 1$, and $K 2$ for GPI; Macintosh et al. [2008]). Therefore, we fit the simulated P1640 spectra as separate $Y J$-band and $H$-band spectra. Results of these fits are discussed in $\S 5.3$ below. Future papers will present this analysis for the exact wavelength coverage and spectral sampling of GPI and SPHERE.

\section{RESULTS}

\subsection{Probability Distributions}

Results from the spectral fitting routine described above are probability distributions with $10^{6}$ values for each parameter. The probability distribution for fits to the simulated spectra from the field M5 spectral template is shown in Figure 5, for the field L1 in Figure 6, for the field L8 in Figure 7, and for the field T4.5 in Figure 8. In all figures the $Y J H$ results are shown in purple, the $Y J$ results in blue, and the $H$ results in red. The lower left panel shows $1-\sigma, 2-\sigma$, and $3-\sigma$ contours of the posterior distribution functions for both parameters, while the top and right panels shows the histograms for temperature and surface gravity, respectively, marginalized over the other parameter.

The probability distributions for the field M5 are presented in Figure 5. The $Y J H$ and $Y J$-band spectra produce similar

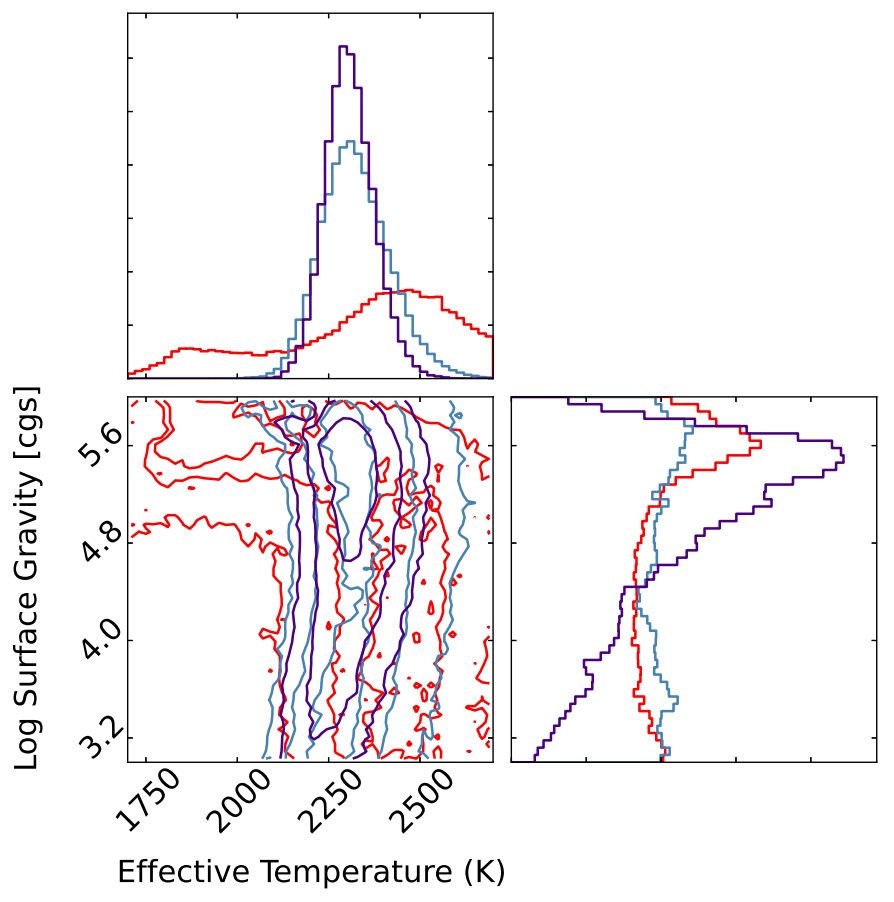

FIG. 6.- Same as Fig. 5 for the field L1 spectral template object. The results for the complete $Y J H$ spectrum are better constrained in temperature than in surface gravity. Temperature is symmetric, while gravity has a longer tail to lower values. $Y J$-band spectra result in a more precise temperature estimate, while $H$-band and the complete $Y J H$ spectrum provide a more reliable gravity estimate as indicated by the sharp decrease in probability at gravities higher than $\sim 5.6$, which are unphysically high but allowed in the model grid, and the less pronounced decrease in probability for $\log (g)<5$.0. See the online edition of the PASP for a color version of this figure.

temperature results, but the $H$-band distribution has broad, flat peak with a long tail toward higher temperatures. None of the distributions shows a clear peak in surface gravity, with the $Y J H$ distribution increasing toward unphysically high surface gravities and the $Y J$ - and $H$-band distributions increasing slightly at values of $\log (g)<4.0$ dex (cgs).

The probability distributions for the field L1 (Fig. 6) are qualitatively similar to the M5 in temperature ( $\sim 600 \mathrm{~K}$ cooler), with the $Y J H$ - and $Y J$-band spectra producing nearly overlapping symmetric and clearly peaked distributions, but the $H$ band distribution has a broad peak at hotter temperatures and low plateau at lower temperatures compared to the $Y J H$ and $Y J$ peaks. In surface gravity, the $Y J H$ - and $H$-band distributions show clear peaks, while the $J$-band distribution is flat. Both the $Y J H$ - and $H$-band spectra show sharp decreases in probability at surface gravities $\geq 5.7$, which are unphysically high values but allowed in the model grid.

The probability distributions for the field L8 are presented in Figure 7. The distribution for the $Y J H$ spectrum is narrow and symmetric in both temperature and gravity. The $Y J$ distribution has a broader, but still single-peaked, distribution in temperature but increases toward unphysical high values in surface 


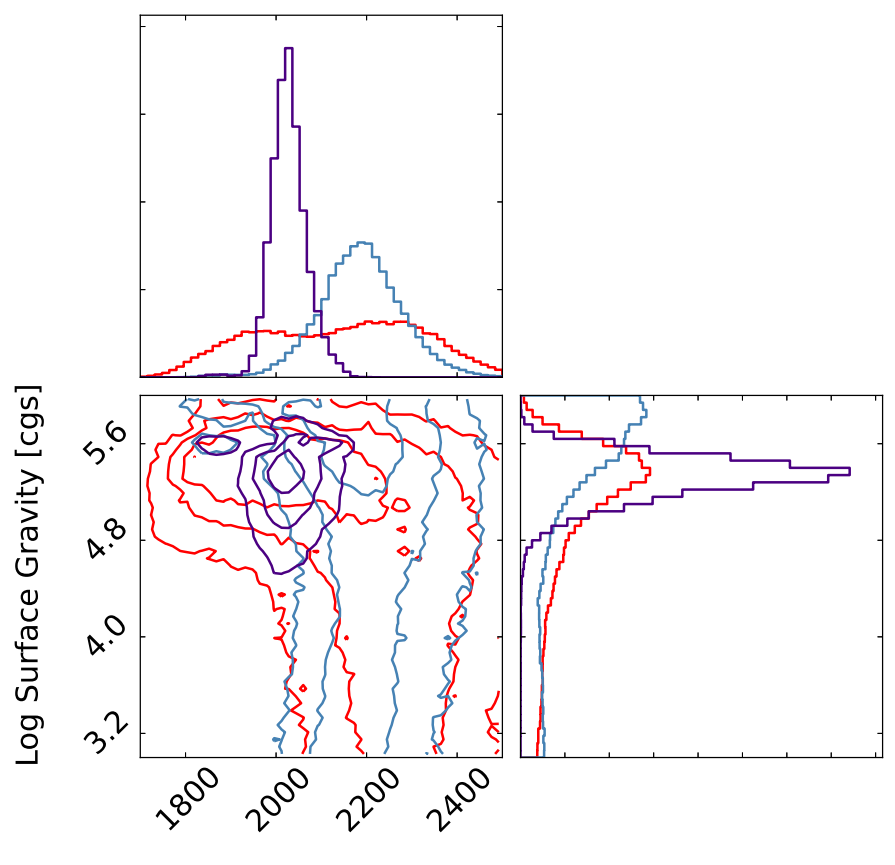

Effective Temperature (K)

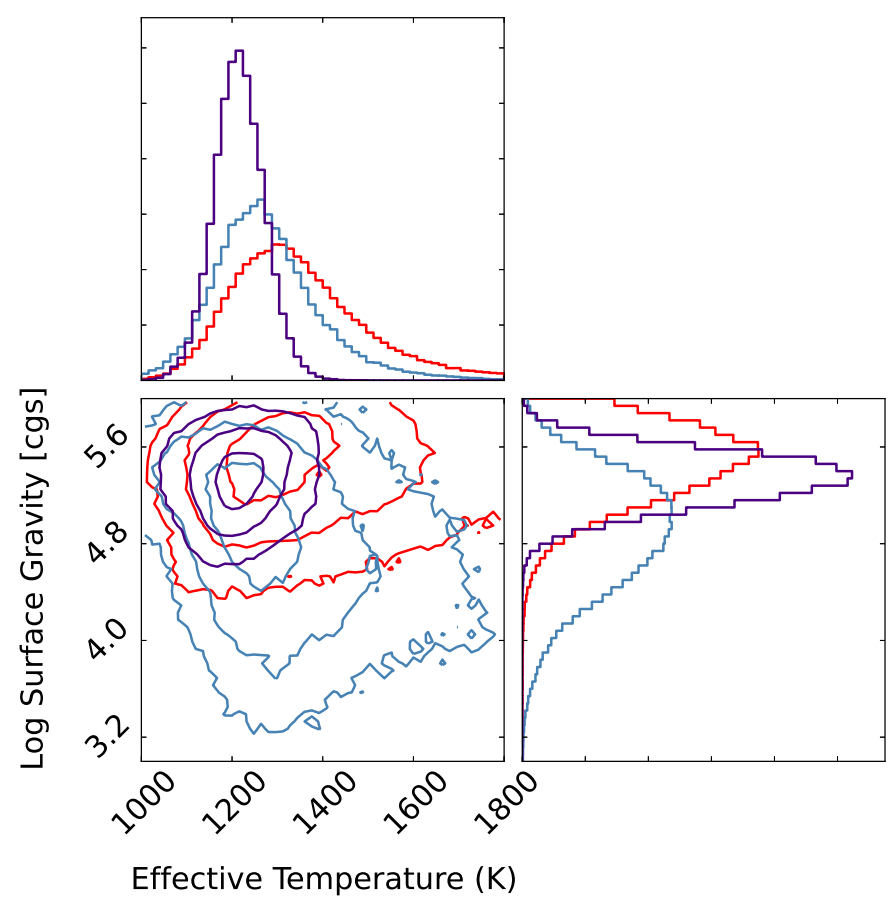

FIG. 8.-Same as Fig. 5 for the field T4.5 spectral template object. The results from different spectral bands are generally consistent, with long tails to higher effective temperatures for the individual $Y J$ - and $H$-band spectra and to lower surface gravities for the $Y J$-band spectrum. See the online edition of the PASP for a color version of this figure.

dusty PHOENIX models are not expected to accurately reproduce cool atmospheres. In surface gravity, the probability distributions also show tighter constraints for later spectral types, although these distributions sometimes extend into unphysical regimes of $\log (g) \geq 5.6$.

\subsection{Best-Fit Spectra}

We adopt the best-fit parameters of the $50 \%$ quantile value for each parameter distribution, which corresponds to the peak of the histograms only for symmetric distributions. The uncertainties on the best-fit parameters are defined by the $16 \%$ and $84 \%$ quantiles of the distributions, which correspond to $1-\sigma$ width for a purely Gaussian posterior. Results are listed in Table 2 and discussed below.

The effective temperature and surface gravity values of the best-fit model spectra for each simulated P1640 spectrum are listed in Table 2, and the corresponding synthetic spectra are overplotted with simulated spectra in Figures 9 and 10. Bestfit parameters were determined via the two-step method described in $\S 4$ above for four versions of the simulated spectra: complete $Y J H$ spectra, "trimmed" $Y J H$ spectra, and separate $Y J$ and $H$ spectra. The best-fit results for the complete and trimmed $Y J H$ spectra are effectively the same (see $\S 5.3$ below) so only the complete $Y J H$ results are reported and plotted, as solid purple lines, in Figures 9 and 10. Blue dashed lines 
TABLE 2

SPECTRAL FitTing Results

\begin{tabular}{|c|c|c|c|c|c|c|}
\hline \multirow[b]{2}{*}{ Spectral type } & \multicolumn{3}{|c|}{$T_{\text {eff }}(\mathrm{K})$} & \multicolumn{3}{|c|}{$\log (g)$} \\
\hline & $Y J H$ & $Y J$ & $H$ & $Y J H$ & $Y J$ & $H$ \\
\hline \multicolumn{7}{|l|}{ Field objects } \\
\hline M1 & $3820_{-444}^{+509}$ & $3866_{-459}^{+418}$ & $3738_{-565}^{+514}$ & $4.85_{-0.83}^{+0.71}$ & $4.55_{-1.00}^{+0.98}$ & $4.58_{-1.02}^{+0.97}$ \\
\hline M5 & $2969_{-135}^{+163}$ & $2934_{-169}^{+239}$ & $3404_{-480}^{+634}$ & $4.76_{-1.06}^{+0.87}$ & $4.35_{-0.99}^{+1.14}$ & $4.41_{-0.93}^{+1.06}$ \\
\hline M8 & $2661_{-100}^{+115}$ & $2639_{-484}^{+169}$ & $2919_{-334}^{+618}$ & $4.70_{-0.93}^{+0.05}$ & $4.41_{-0.98}^{+1.07}$ & $4.30_{-0.94}^{+1.19}$ \\
\hline L1 & $2305_{-62}^{+69}$ & $2317_{-86}^{+100}$ & $2433_{-353}^{+259}$ & $5.08_{-0.98}^{+0.93}$ & $4.55_{-1.08}^{+1.08}$ & $4.73_{-1.23}^{+0.94}$ \\
\hline L5 & $2098_{-44}^{+49}$ & $2159_{-83}^{+86}$ & $2229_{-355}^{+183}$ & $5.35_{-0.38}^{+0.28}$ & $4.98_{-1.29}^{+0.08}$ & $4.94_{-1.17}^{+0.59}$ \\
\hline L8 & $2026_{-34}^{+39}$ & $2187_{-86}^{+87}$ & $2149_{-217}^{+180}$ & $5.33_{-0.21}^{+0.16}$ & $5.38_{-1.41}^{+0.45}$ & $5.11_{-1.08}^{+0.40}$ \\
\hline $\mathrm{T} 2$ & $1663_{-92}^{134}$ & $1374_{-116}^{+194}$ & $1782_{-150}^{+151}$ & $5.21_{-0.21}^{+0.21}$ & $5.45_{-0.41}^{+0.41}$ & $5.20_{-0.30}^{+0.08}$ \\
\hline $\mathrm{T} 4.5$ & $1215_{-53}^{+55}$ & $1264_{-96}^{+119}$ & $1328_{-120}^{+168}$ & $5.34_{-0.23}^{+0.21}$ & $4.91_{-0.52}^{+0.45}$ & $5.48_{-0.36}^{+0.29}$ \\
\hline \multicolumn{7}{|l|}{ Young objects } \\
\hline M8.5 $\gamma$ & $2246_{-57}^{+62}$ & $2337_{-90}^{+105}$ & $2347_{-571}^{+220}$ & $5.70_{-0.35}^{+0.18}$ & $4.30_{-0.94}^{+1.21}$ & $4.34_{-1.00}^{+1.18}$ \\
\hline $\mathrm{L} 0 \gamma$ & $2093_{-88}^{+97}$ & $2205_{-85}^{+100}$ & $2239_{-544}^{+353}$ & $5.89_{-0.10}^{+0.07}$ & $4.03_{-0.76}^{+1.53}$ & $4.74_{-1.37}^{+0.94}$ \\
\hline $\mathrm{L} 5 \gamma$ & $1780_{-26}^{+32}$ & $1969_{-161}^{+116}$ & $1634_{-59}^{+674}$ & $5.29_{-0.27}^{+0.21}$ & $5.01_{-1.33}^{+0.70}$ & $4.80_{-0.75}^{+0.52}$ \\
\hline L7 VL-G & $1739_{-23}^{+31}$ & $1947_{-138}^{+89}$ & $1659_{-58}^{+65}$ & $5.02_{-0.30}^{+0.20}$ & $5.44_{-0.75}^{+0.39}$ & $4.65_{-0.72}^{+0.47}$ \\
\hline
\end{tabular}

NOTE.-Best-fit parameters are $50 \%$ quantile values, and quoted uncertainties are derived from $16 \%$ and $84 \%$ quantiles, equivalent to $1-\sigma$ uncertainties for Gaussian posterior distributions (see § 5.1).
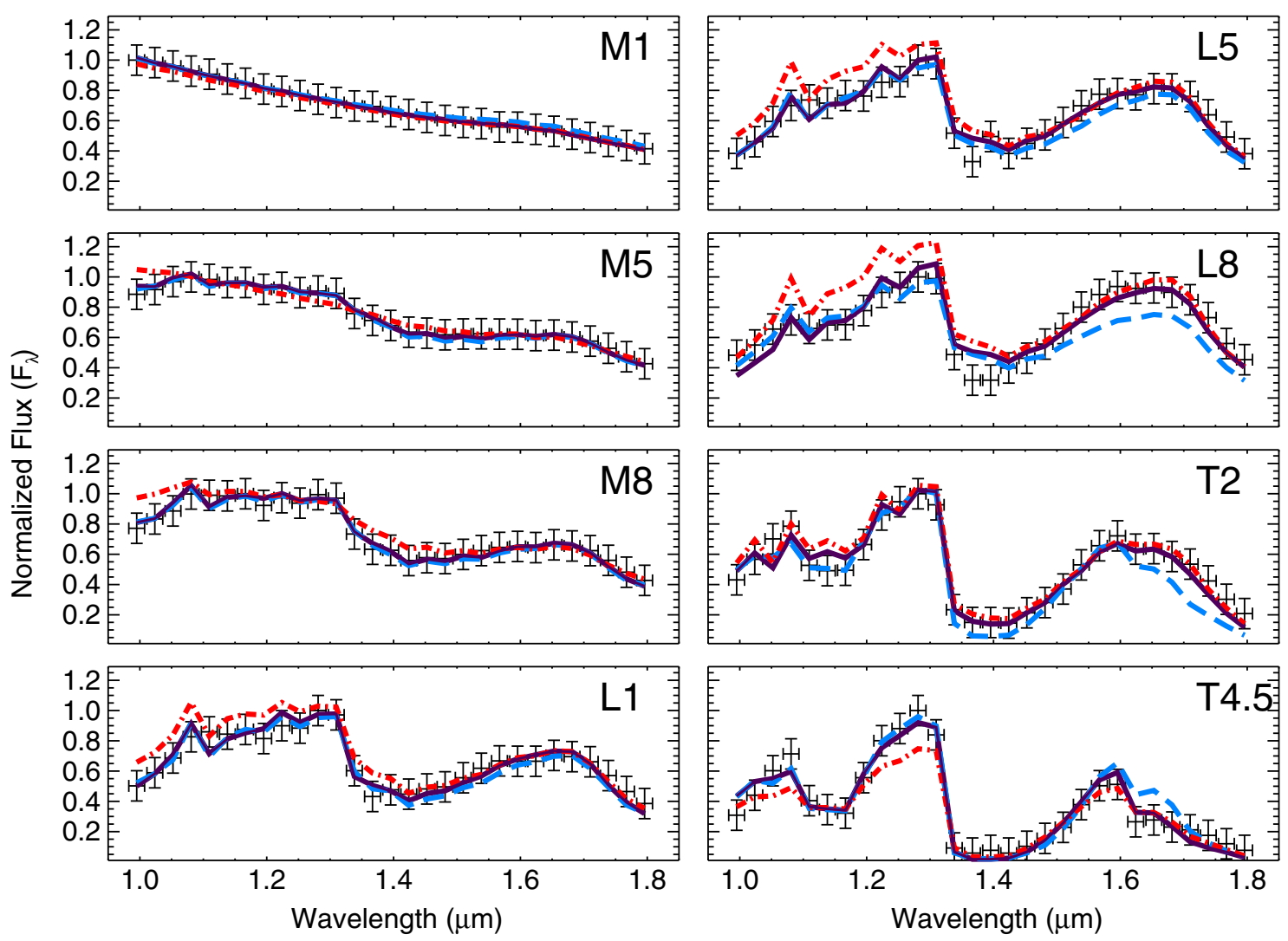

FIG. 9.-Best-fit PHOENIX model spectra for simulated P1640 spectra (gray error bars) of field M, L, and T dwarfs. Vertical error bars represent the constant noise value with $\mathrm{S} / \mathrm{N}=10$ at the peak flux, and horizontal errors bars represent the width of one wavelength channel. Purple lines represent the best-fit model spectrum using all flux points in the simulated spectra except four points in the $\mathrm{H}_{2} \mathrm{O}$ band $\left.1.34-1.42 \mu \mathrm{m}\right)$. Blue dashed lines represent the best-fit model spectra using just the $\sim Y J$-band flux points $(0.995-1.31 \mu \mathrm{m})$, and red dot-dashed lines the $H$-band flux points $(1.45-1.80 \mu \mathrm{m})$. M and L dwarf fits use the dusty version of the PHOENIX models, and T dwarf fits use the cond version. See the online edition of the PASP for a color version of this figure. 

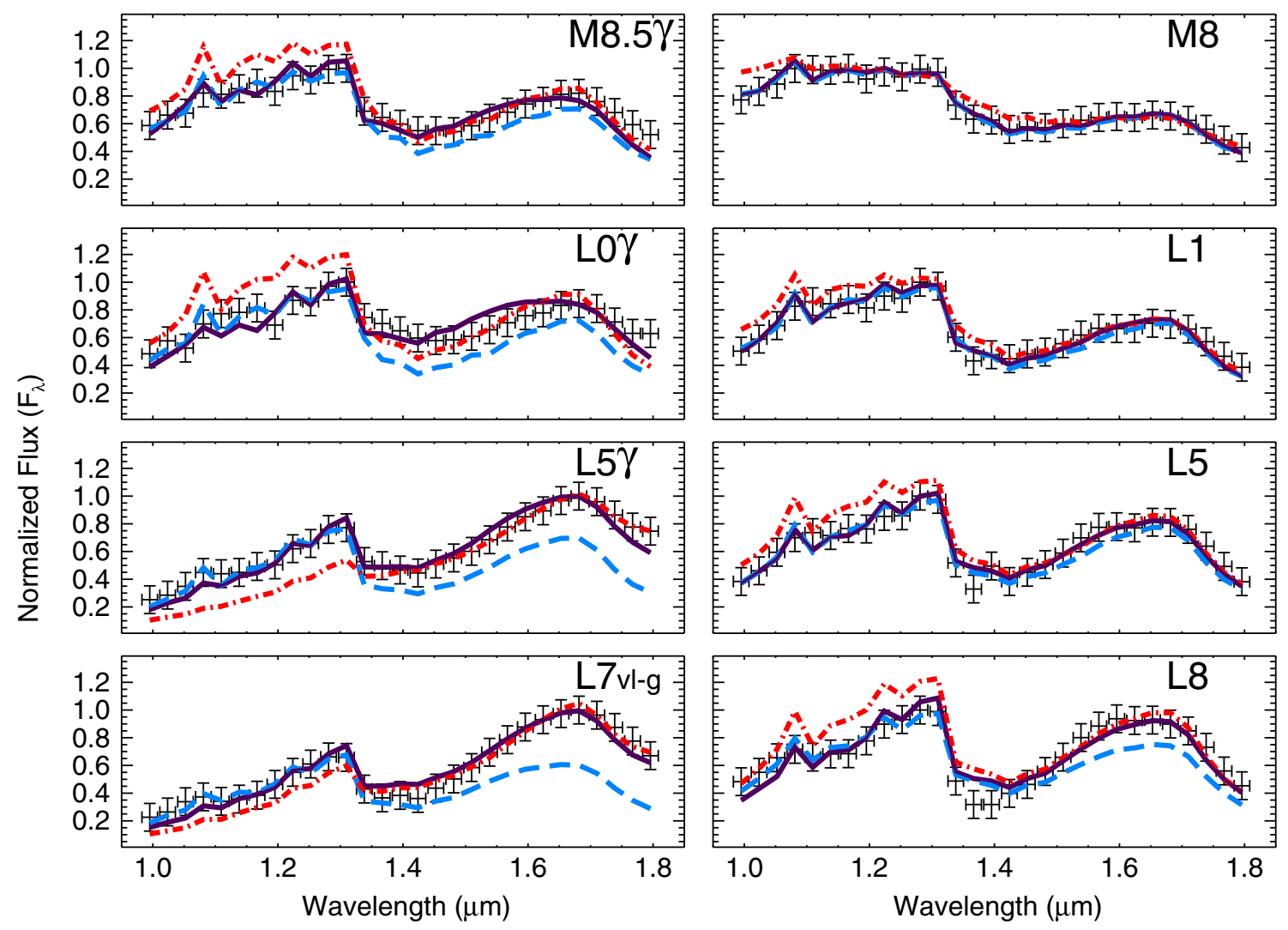

FIG. 10.-Best-fit PHOENIX dusty model spectra for simulated P1640 spectra (gray error bars) of young M and L spectral type objects (left) and their closest field-age counterparts in our sample (right). Vertical error bars represent the constant noise value with $\mathrm{S} / \mathrm{N}=10$ at the peak flux, and horizontal errors bars represent the width of one wavelength channel. Purple lines represent the best-fit model spectrum using all flux points in the simulated spectra except four points in the $\mathrm{H}_{2} \mathrm{O}$ band 1.34-1.42 $\mu \mathrm{m}$. Blue dashed lines represent the best-fit model spectra using just the $\sim Y J$-band flux points $(0.995-1.31 \mu \mathrm{m})$ and red dot-dashed lines the $H$-band flux points $(1.45-1.80 \mu \mathrm{m}) . \mathrm{M}$ and $\mathrm{L}$ dwarf fits use the dusty version of the PHOENIX models. See the online edition of the PASP for a color version of this figure.

represent the best-fit synthetic spectrum with model parameters determined using only the $Y J$-band flux points $(0.995-1.31 \mu \mathrm{m})$, and red dot-dashed lines represent the $H$-band flux points $(1.45-1.80 \mu \mathrm{m})$. All three best-fit spectra are plotted over the complete $Y J H$ wavelength range of the simulated spectra.

The best-fit synthetic spectra from the $Y J H$ fits reproduce the overall spectral shape and broad absorption features, within the assigned error bars of the simulated spectra, for all spectral types. The only marginal exceptions are 1-2 points in the trough of the $1.4 \mu \mathrm{m} \mathrm{H}_{2} \mathrm{O}$ absorption band for the field L5 and L8 objects, one point in the $Y$-band peak of the T4.5 dwarf, as well as two points around $1.4 \mu \mathrm{m}$ and the slope on either side of the $\mathrm{H}$ band peak for the $\mathrm{L} 0 \gamma$ object. This is remarkable especially considering the limiting case dust treatments of the PHOENIX dusty and cond models and the discrepancy of the model parameters with previous measurements from the literature ( $\$ \S 5.4$ and 5.5). Therefore, we advise that even good fits should be treated with caution when interpreting fits to low-resolution near-infrared spectra for very low-mass objects.

The fits to different subsets of the flux points, based on traditional filters separated by the telluric absorption band at
$1.4 \mu \mathrm{m}$, test the consistency of the best-fit parameters from these different bands. For the $\mathrm{M}$ dwarfs, all of the fits produce similar results, with the $H$-band fit slightly over predicting the first two or three $Y$-band flux points for the M5 and M8. For the $\mathrm{L}$ dwarfs, the fits become less consistent, with the $H$-band parameters over predicting the flux for the $Y J$-band points and the $Y J$-band fit under predicting the flux at $H$-band, especially for the L8 object. The T dwarfs are slightly better, with the only problems being the $Y J$ fits underpredicting the red end of the $H$-band for the $\mathrm{T} 2$ and slightly overpredicting the flux for the T4.5, and the $H$-band fit slightly underpredicting the $Y$ - and $J$-band peaks for the T4.5.

The offsets between the synthetic spectra from $Y J$ - or $H$ band fits and the rest of the simulated spectra are more significant for the young objects, particularly for the low-gravity L spectral types. Figure 10 shows the best-fit spectra for the young objects (left) with the best-fit spectra for field objects of similar spectral types (right). For the hotter objects (M8.5 $\gamma$ and $\mathrm{L} 0 \gamma$ ) the $Y J$-band fits only slightly underpredict the $H$-band flux points (blue dashed line), while the $H$-band fits are more significantly different, especially for the $\mathrm{L} 0 \gamma$ spectrum (red 
dot-dashed line). The single-band best-fit spectra for the cooler objects (L5 $\gamma$ and L7 VL-G) always underpredict the flux in the other band, most substantially for the $Y J$-band fit in the $H$ band (blue dashed line).

Overall, the low-resolution fits to the complete $Y J H$ spectra reproduce the simulated low-resolution near-infrared spectra for all spectral types, despite using atmosphere models with limiting cases of dust treatment to produce the synthetic spectra. Fits to an individual bands result in significant discrepancies with flux points in the other band, suggesting that a broad wavelength coverage is best for reliable characterization of low-mass companions via low-resolution spectra.

\subsection{Effect of Trimming Spectra}

The $\mathrm{H}_{2} \mathrm{O}$ absorption band at $\sim 1.4 \mu \mathrm{m}$ has a typically low $\mathrm{S} / \mathrm{N}$ and is most likely to be affected by systematic errors induced by telluric calibration, which are difficult to quantify. These wavelength points are typically excluded from analysis for observed P1640 spectra (e.g., Roberts et al. 2012; Oppenheimer et al. 2013). Therefore, we fit "trimmed" simulated P1640 spectra by excluding four flux points in the $\sim 1.4 \mu \mathrm{m}$ $\mathrm{H}_{2} \mathrm{O}$ absorption band to determine the effect of removing these flux values on the best-fit model parameters.

The resulting best-fit parameters were identical within the uncertainties in both cases, with the largest differences being $15 \mathrm{~K}$ for the M5 object and 0.15-0.25 dex for the field L5 and L8 objects and the young M8.5 $\gamma$ object. The temperature difference is 10 times smaller than the $1-\sigma$ uncertainty defined by the $16 \%$ and $84 \%$ quantile values. The gravity differences are comparable to the $1-\sigma$ uncertainties for those objects. Uncertainties derived from the probability distributions were also very similar, within $15 \mathrm{~K}$ and $0.04 \operatorname{dex} \log (g)$, except for the $\log (g)$ of the M8.5 $\gamma$ object, with the $1-\sigma$ quantile extending 1.4 dex lower in surface gravity for the trimmed spectrum.

These results suggest that excluding low $\mathrm{S} / \mathrm{N}$ flux measurements in the water band have little effect on the parameters of very low-mass objects inferred from model fits to low-resolution near-infrared spectra, but they might be marginally significant for hotter low-gravity objects. Results for the young objects are discussed in more detail in $\S 5.6$ below.

\subsection{Effective Temperature}

Figure 11 shows the effective temperatures of the best-fit synthetic spectra as a function of spectral type for the field objects (filled symbols) and young objects (open symbols) for each of the three versions of the spectra: $Y J$ (upward blue triangle), $H$ (downward red triangle), and complete YJH (purple square). The triangle points are offset slightly in spectral type for clarity. The gray shaded region shows temperatures derived for the same spectral types from Luhman \& Rieke (1999) for M dwarfs and Stephens et al. (2009) for L and T dwarfs with a range of $\pm 100 \mathrm{~K}$.

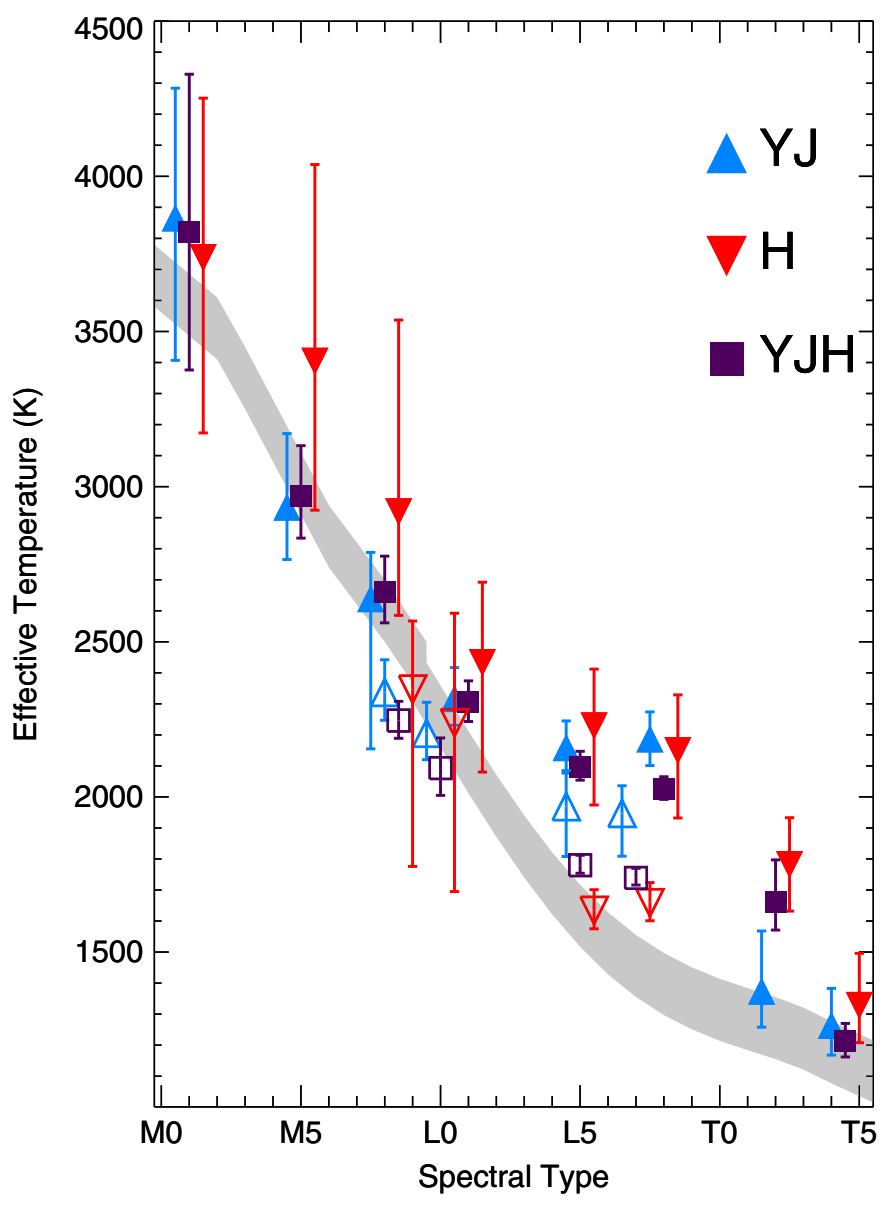

FIG. 11.-Best-fit results in effective temperature versus spectral type for simulated P1640 data fit as $Y J$-band (blue upward triangles), $H$-band (red downward triangles) and $Y J H$-band (purple squares) spectra. Field objects are represented by filled symbols and young objects, by open symbols. The gray filled region shows temperatures derived for objects with the same spectral types from Luhman \& Rieke (1999) for M dwarfs and Stephens et al. (2009) for L and T dwarfs with a range of $\pm 100 \mathrm{~K}$. See the online edition of the PASP for a color version of this figure.

For model fits to low-resolution near-infrared spectra to be considered reliable, they should be consistent with the physical parameters derived from other methods. Figure 11 shows that the best-fit results from low-resolution near-infrared spectra (symbols) match the empirically derived temperatures (gray shaded regions) to within the uncertainties for the field $\mathrm{M}$ dwarfs and the T4.5 dwarf, but the model fits result in hotter temperature for the field L5, L8, and T2 dwarfs, by as much as $500 \mathrm{~K}$ (for the L8 dwarf). This is the temperature regime in which dust is expected to condense from the photosphere, a dynamic and complex process that is not modeled in detail with the limiting cases of dust treatment in the PHOENIX dusty and cond model atmospheres.

The best-fit effective temperatures from different segments of the spectra are generally consistent for a given object within the 
$1-\sigma$ uncertainties, with notable exceptions of the $Y J$ fits for the $\mathrm{L} 7 \mathrm{VL}-\mathrm{G}, \mathrm{L} 8$, and $\mathrm{T} 2$ objects. The $H$-band fits produce the highest temperatures for almost all of the field objects and lower temperatures for the young L dwarfs.

Uncertainties in effective temperature are large (up to $\pm 500 \mathrm{~K}$ ) for the earliest spectral types considered in our analysis, but these are still 10-20\% precision. Statistical uncertainties are as small as $\pm 30-50 \mathrm{~K}$ for the mid- to late-L dwarfs (field and young), although these values are also systematically hotter than the empirically derived temperatures, particularly for the field objects. As might be expected, the uncertainties are smallest for the complete $Y J H$ spectra, but in some cases the uncertainties on individual bands are nearly as small, particularly for the $Y J$-band of the M8.5 $\gamma, \mathrm{L} 0 \gamma, \mathrm{L} 5$, and $\mathrm{L} 7$ objects and for the $H$-band of the L5 $\gamma$, L7 VL-G, and T2 objects.

The best-fit effective temperature for known young objects are shown on Figure 11 as open symbols. All young object spectral fits produce cooler temperature best-fits than corresponding fits to spectra of field objects with similar spectral types. This is consistent with the idea that enhanced dust in cool, low-gravity atmospheres causes the near-infrared spectrum to appear more red (i.e., cooler) than would be predicted by the optically defined spectral type (e.g., Metchev \& Hillenbrand 2006; Luhman et al. 2007; Barman et al. 2011b; Bowler et al. 2013). Indeed, direct empirical comparison of bolometric luminosities for young and field objects of the same spectral types suggest that, because they are similar but the young objects should have inflated radii, the young objects must have lower effective temperatures than their field-age spectral type counterparts (J. C. Filippazzo et al., in preparation).

\subsection{Surface Gravity}

Substellar-mass objects never reach a stable main sequence; instead they cool, fade, and shrink for their entire lifetimes. Effective temperature and luminosity are degenerate with age and mass, but surface gravity is uniquely and significantly low $(<5.0$ dex cgs) for young objects $(<100 \mathrm{~s} \mathrm{Myr})$. We include four known young substellar objects in our sample to test whether model fits are sensitive enough to surface gravity to distinguish young from old objects, or even to constrain age via comparison with evolutionary models, using low-resolution near-infrared spectra.

Figure 12 shows the surface gravity of the best-fit model as a function of spectral type with the same symbols as in Figure 11 described in $\S 5$.4. The shaded regions represent predictions for field (age $\geq 500 \mathrm{Myr}$, solid) and young (age 5-120 Myr, hatched) objects from evolutionary models: Siess et al. (2000) for $T_{\text {eff }}>3000 \mathrm{~K}$, Baraffe et al. (2002) and Chabrier et al. (2000) [DUSTY00] for $3000>T_{\text {eff }}>1500 \mathrm{~K}$, and Baraffe et al. (2003) [COND0] for $T_{\text {eff }}<1500 \mathrm{~K}$. The hatched gray regions shows predictions from the DUSTY00 models for effective temperatures $\sim 2500-1500 \mathrm{~K}$, which approximately corresponds to the spectral types of young objects in our sample.

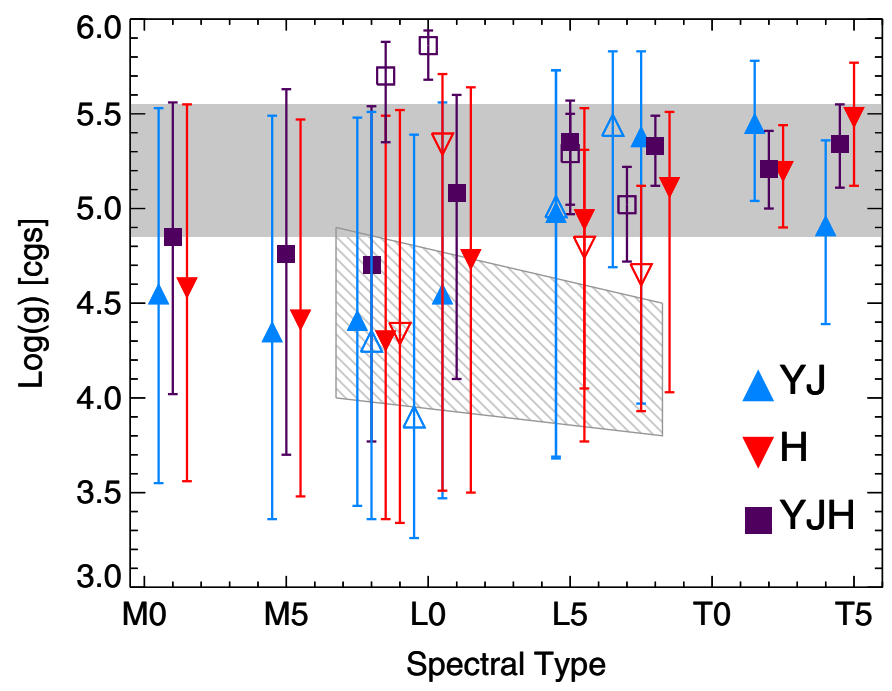

FIG. 12.-Best-fit results in surface gravity versus spectral type for simulated P1640 data fit as $Y J$-band (blue upward triangles), $H$-band (red downward triangles) and $Y J H$-band (purple squares) spectra. Field objects are represented by filled symbols and young objects, by open symbols. The solid shaded region shows the range of surface gravities for field objects (age $\geq 500 \mathrm{Myr}$ ) predicted by evolutionary models: Siess et al. (2000) for $T_{\text {eff }}>3000 \mathrm{~K}$, Baraffe et al. (2002) and Chabrier et al. (2000) [DUSTY00] for $3000>T_{\text {eff }}>1500 \mathrm{~K}$, and Baraffe et al. (2003) [COND03] for $T_{\text {eff }}<1500 \mathrm{~K}$. The hatched gray region shows predictions from the DUSTY00 models for effective temperatures $\sim 2500-1500 \mathrm{~K}$, which corresponds approximately to the spectral types of young objects in our sample. The surface gravity results for field objects are closer to predictions from evolutionary models and more consistent between individual wavelength regions than the results for the young objects. See the online edition of the PASP for a color version of this figure.

Broadly, evolutionary models predict the surface gravity for field early-M dwarfs to be in the lowest part of the shaded parameter range on Figure 12, i.e., $\log (g)=4.9$ dex. The oldest late-L dwarfs are predicted to peak at $\log (g)=5.4 \mathrm{dex}$, and the oldest, most massive T dwarfs reach $\log (g)=5.5$ dex. For substellar-mass objects, younger objects will have lower surface gravities as a result of their larger radii, but objects older than $500 \mathrm{Myr}$ will have $\log (g)>4.9$ for the entire range of temperatures considered, according to both the DUSTY00 and COND03 evolutionary models.

The best-fit surface gravities are consistent with the values predicted by evolutionary models for field objects of all spectral types, within the relatively large $1-\sigma$ uncertainties (from \pm 0.2 to $\pm 0.9 \mathrm{dex})$. For the $\mathrm{M}$ and early-L dwarfs, the $Y J H$ best-fit surface gravity is closer to the predicted value than the surface gravities from individual $Y J$ - and $H$-band fits. For field $\mathrm{L}$ and $\mathrm{T}$ dwarfs, the best-fit surface gravity is within the range of values predicted by the evolutionary models, except for the individual $Y J$ - and $H$-bands for the $\mathrm{L} 1$ object. This is likely a result of the broad spectral features being increasingly shaped by surface gravity, in addition to temperature, for the coolest objects. It is perhaps surprising that such low-resolution spectra are sensitive to surface gravity at all, but it has been shown that the 
relative strengths of broadband absorption like $\mathrm{H}_{2} \mathrm{O}$ and collisionally induced absorption from $\mathrm{H}_{2}$ depend on surface gravity (Fig. 6 from Rice et al. [2011]).

The results from fits using different wavelength ranges for field objects are generally self-consistent to within the $1-\sigma$ uncertainties. The largest discrepancy is for the field L5, where the individual $Y J$ - and $H$-band fits indicate lower surface gravities ( $\sim 5.0 \mathrm{dex}$ cgs) and the complete $Y J H$ fit indicates a higher surface gravity $(5.35 \mathrm{dex} \operatorname{cgs})$. The three fits indicate similar effective temperatures $(\sim 2100-2230 \mathrm{~K})$, with the individual bands at the hotter end of the range. The results are all within the $1-\sigma$ uncertainties for each parameter so are not likely to indicate any systematic trend.

The results for best-fit surface gravity for the young objects are more discrepant from predictions of the evolutionary models and less consistent for fits from individual wavelength ranges. They are discussed in more detail in $\S 5.6$ below.

\subsection{Young Objects}

The primary targets of exoplanet direct-imaging campaigns are nearby young stars. Planetary-mass companions to young stars are more luminous than older objects because they are still contracting and radiating gravitational potential energy. Young brown dwarfs are similarly low-mass and low-surface-gravity, but as free-floating objects they are amenable to a broader range of observations, both in wavelength coverage and spectral resolution. Therefore, young brown dwarfs are potentially important analogs to directly imaged exoplanets (see, e.g., Faherty et al. 2013; Allers \& Liu 2013).

We include four confirmed young brown dwarfs in our analysis in order to test whether model spectra reproduce the lowresolution near-infrared spectra of cool, low-gravity objects and to compare the accuracy and consistency of best-fit results for young and field objects with similar spectral types. The young template objects we use have optical or near-infrared spectral types of M8.5 $\gamma$, L0 $\gamma$, L5 $\gamma / \mathrm{L} 3$ VL-G, and L7 VL-G (see Cruz et al. [2009] and Allers \& Liu [2013] for descriptions of the surface gravity suffxes) and medium-resolution, high S/N spectra available in the literature (see Table 1 for references).

Figure 13 shows the posterior probability distribution functions for young M8.5 $\gamma$ and L5 $\gamma$ objects and the field objects with the closest spectral types, with the $Y J H$ results in purple, the $Y J$ results in blue, and the $H$ results in red. The scales on each axis are identical in effective temperature and log surface gravity in order to show systematic difference between results for objects on the same optically defined spectral type but difference ages (field objects in top row and young objects in bottom row). For both young objects, the posterior distribution functions are centered on significantly cooler temperatures $\left(\Delta T_{\text {eff }}=300-600 \mathrm{~K}\right)$ than for the comparable field object. For the young M8.5 $\gamma$ object, the $Y J H$ and $Y J$ fits are consistent in temperature, but the $H$-band fit is bimodal with the a significantly cooler component that is a very poor fit to the
$Y J$ spectrum. For the young L5 $\gamma$ object, the complete $Y J H$ spectrum temperature distribution is consistent with the $Y J$ and $H$ distributions to within $1-\sigma$.

Figure 14 shows the posterior probability distributions for model fits to the L7 VL-G spectra. The distributions from fits to different spectral regions are substantially less consistent than for the field objects (Figs. 5-8). For example, the $2-\sigma$ contours of the $Y J$-band (blue) and $H$-band (red) distributions just barely overlap. The $H$-band distribution peaks at a surface gravity predicted for a young, late-type object (see Fig. 12), but the $Y J H$ - and $Y J$-band distributions peak at higher surface gravities and have only a low tail toward lower values. This is further evidence that the PHOENIX dusty models do not accurately reproduce the observed spectra of young, late-type objects (e.g., Schmidt et al. 2008; Mohanty et al. 2010; Patience et al. 2012).

Figure 10 shows the simulated and best-fit spectra for the young objects (left) along with simulated and best-fit spectra for field objects of similar spectral types (right). The model spectra with the $Y J H$ best-fit parameters are plotted as solid purple lines, the $Y J$-band fit as dashed blue lines, and the $H$-band fit as dot-dashed red lines. For all four young objects, the complete (and trimmed) best-fit $Y J H$ spectrum reproduces the simulated spectrum to within the adopted $>10 \%$ uncertainties; however, the best-fit parameters do not match predictions from evolutionary models, as discussed below.

The field M8 and young M8.5 $\gamma$ have similar optically determined spectral types, but the $Y J H$ spectrum of the young object has a more steeply sloped $Y J$-band, deeper $\mathrm{H}_{2} \mathrm{O}$ absorption, and a higher, more triangular $H$-band peak. Fits to the complete $Y J H$ spectra are of similar quality, but fits to different wavelength ranges are less consistent for the young object than for its field-age counterpart. For the young object, the $Y J$-band fit slightly underpredicts the flux throughout the $H$-band, and even the $H$-band fit is slightly lower than the object's flux beyond $1.7 \mu \mathrm{m}$. The $H$-band fit overpredicts the $Y J$-band flux even more, well outside of the adopted flux uncertainties. This is qualitatively similar to single-band results for the field L5 and L8 dwarfs, which are likely the dustiest of the field objects.

The effective temperatures of the M8.5 $\gamma$ best-fit spectra are 400-600 K cooler than those for the field M8 (Fig. 11). The best-fit surface gravity for the young M8.5 $\gamma$ is comparable to that of the and field object in $Y J$ - and $H$-bands and match predictions from the DUSTY00 evolutionary models for 5120 Myr objects (gray hatched region on Fig. 12). The bestfit surface gravity for the $Y J H$ best-fit spectrum is 1.0 dex (cgs) more than that of the field object, and is in fact higher than the maximum value predicted by evolutionary models for any substellar object (e.g., Chabrier et al. 2000; Baraffe et al. 2002, 2003).

The complete $Y J H$ fits to the simulated spectra of young $\mathrm{L}$ spectral types generally reproduce the flux points within the $1-\sigma$ error bars, except for the longest wavelength $H$-band point for three objects and several $J$-band and $H$-band points 


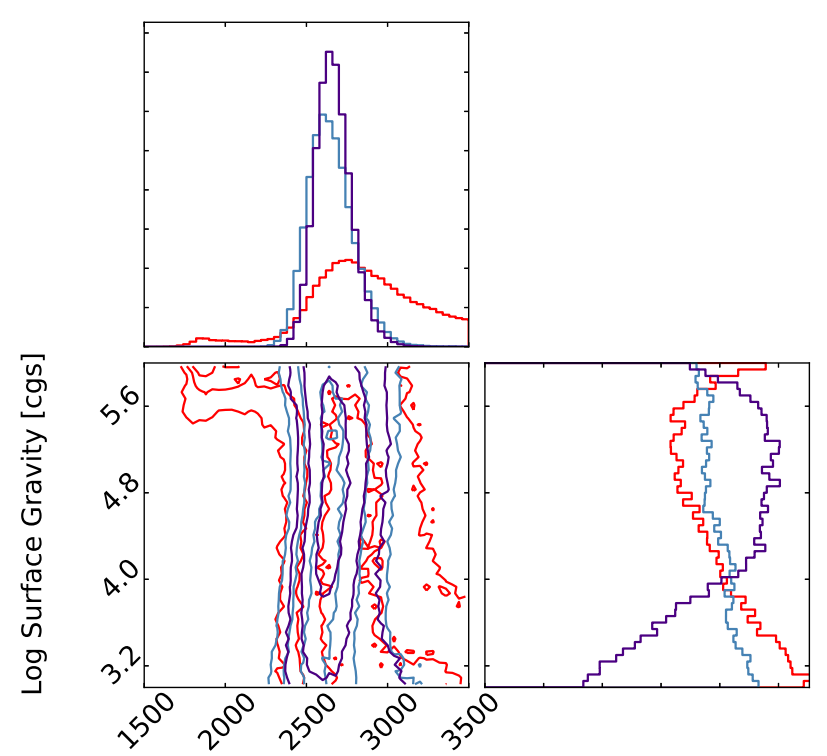

Effective Temperature (K)

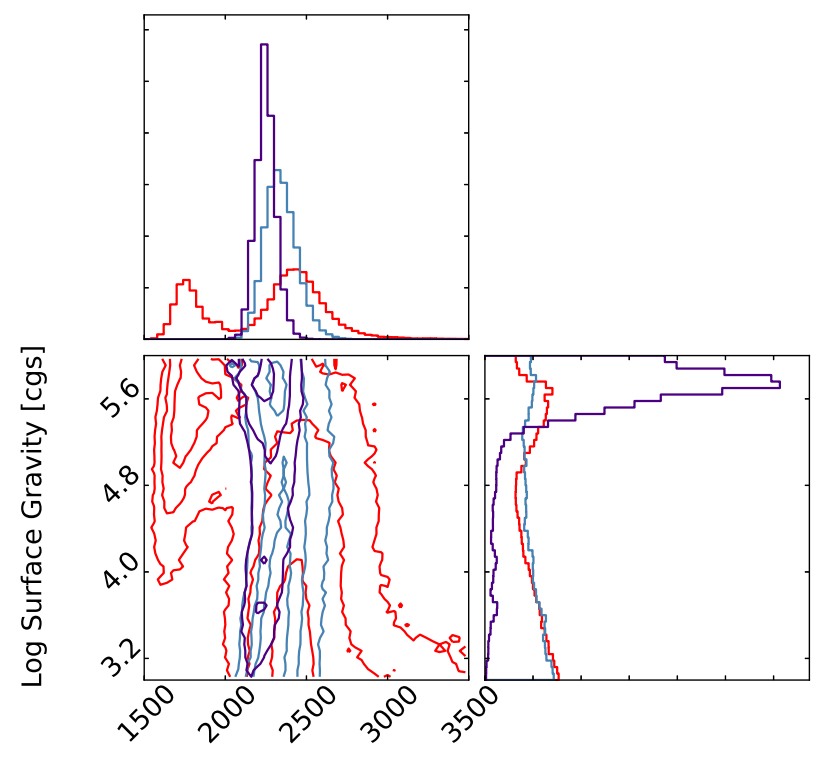

Effective Temperature (K)

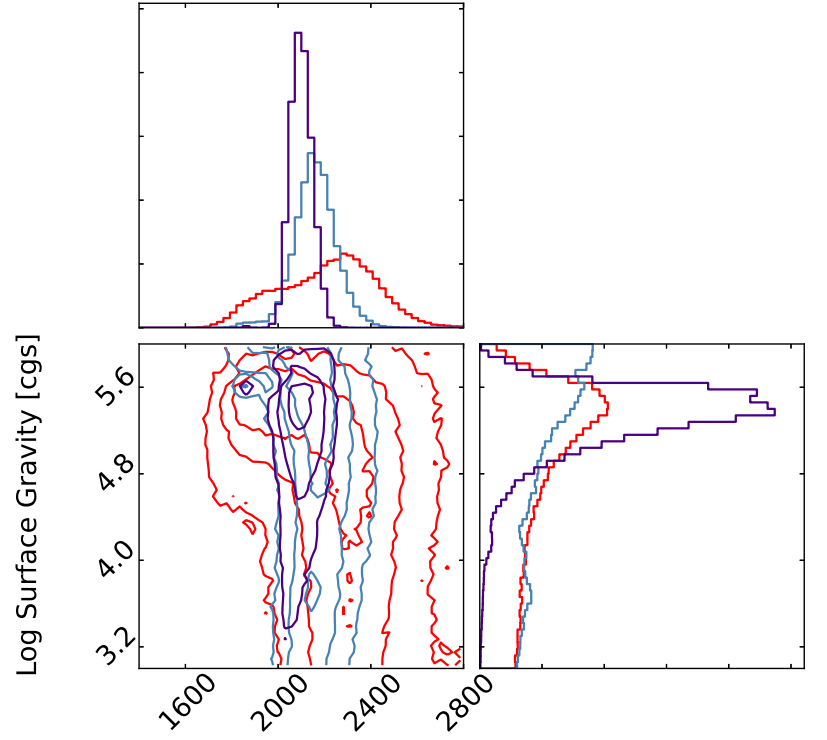

Effective Temperature (K)

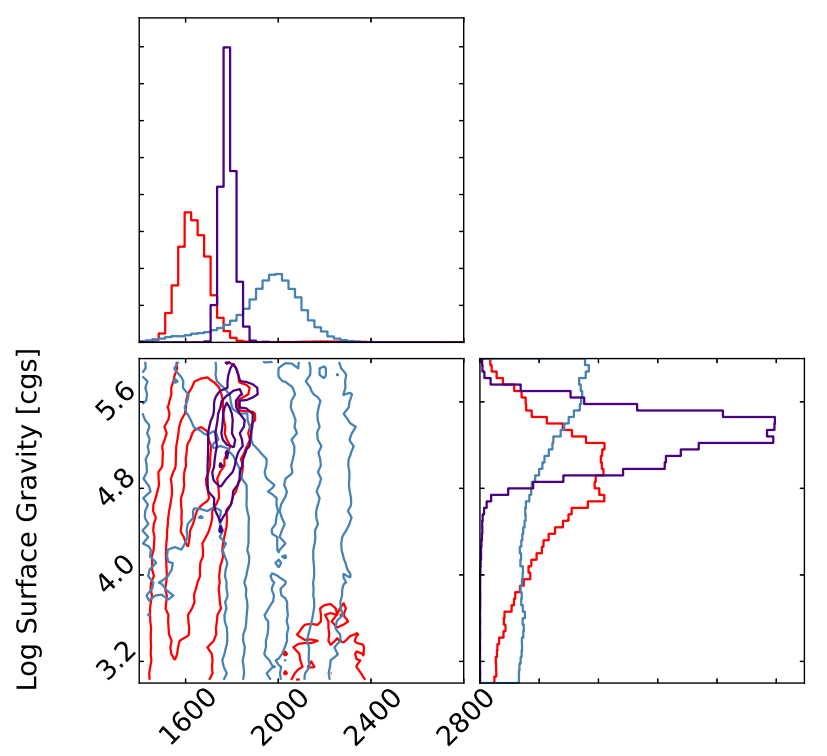

Effective Temperature (K)

FIG. 13.-MCMC results for young and field objects of approximately the same spectral type, M8 objects (left) and L5 objects (right), field objects (top) and young objects (bottom). Colors correspond to different spectral fits as in Fig. 5. The peak of the distributions in effective temperature for the young objects are cooler than the corresponding peak for the field objects. Both young objects also have distributions that peak at higher surface gravities than predicted by evolutionary models using ages constrained by their likely membership in nearby young moving groups. See $\S 5.6$ for a complete discussion of the results. See the online edition of the $P A S P$ for a color version of this figure.

for the $\mathrm{L} 0 \gamma$ object. For the $\mathrm{L} 0 \gamma$ object, the $Y J H$ fit appears overall too flat at the blue end of the $H$ band, which corresponds to a high best-fit surface gravity. The inconsistency between fits for individual bands is even more substantial for the young $\mathrm{L}$ objects than for field L dwarfs. For all three objects, the individual $Y J$ - and $H$-band fits under predict the flux in the other band. The worst $H$-band fit in the $Y J$-band is for the $\mathrm{L} 0 \gamma$, while the worst $Y J$-band fit in the $H$-band is for the $\mathrm{L} 7 \mathrm{VL}$-G object, which is the reddest of the young $\mathrm{L}$ dwarfs.

All fits for the young Ls produce lower effective temperatures than for the field objects of similar spectral type, especially for the $Y J H$ - and $H$-band fits (Fig. 11). The best-fit surface gravities for the $\mathrm{L} 0 \gamma$ are similar to that of the $\mathrm{M} 8.5 \gamma$ in that the best-fit parameters from the $Y J H$ fits are too high, but 


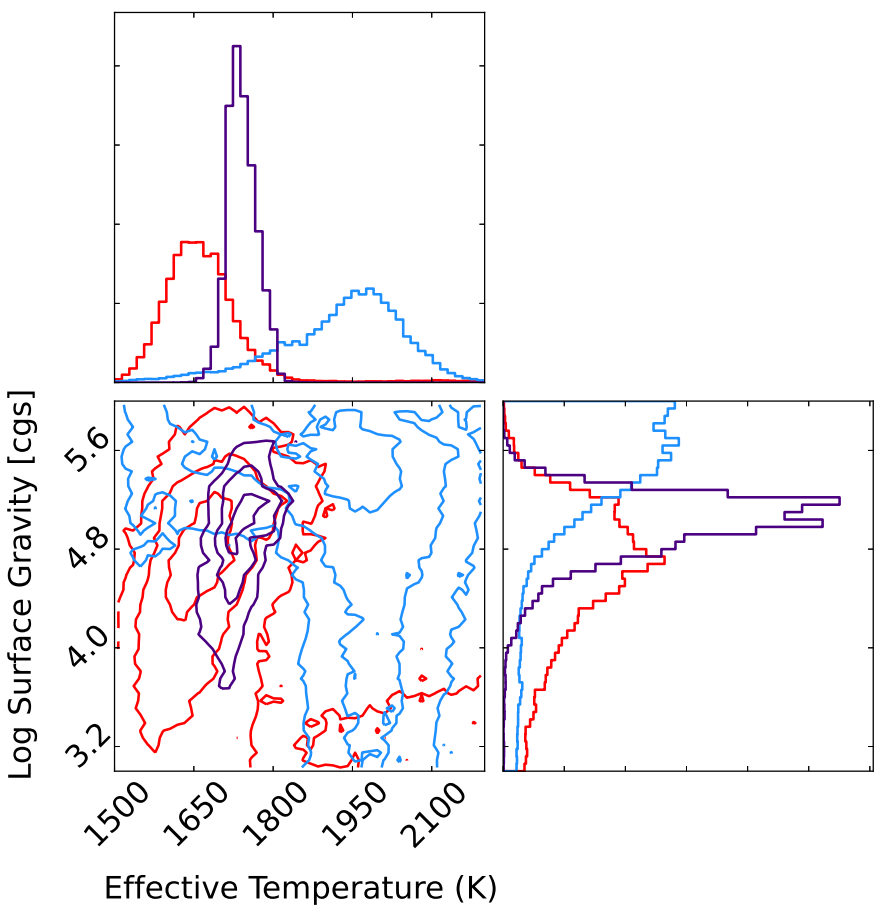

FIG. 14.-Same as Fig. 5 for the young L7 VL-G object. The distributions from different spectral regions are substantially less consistent than for the field objects. For example, the $2-\sigma$ contours of the $Y J$-band (blue) and $H$-band (red) distributions just barely overlap. While, the $H$-band distribution peaks at a surface gravity predicted for a young, late-type object (see Fig. 12), the $Y J H$ - and $Y J$-band distributions are peaked at higher surface gravities and have only a long tail toward lower values. See the online edition of the PASP for a color version of this figure.

the best-fit parameters from the $Y J$ - and $H$-band fits are comparable to predictions from the DUSTY00 evolutionary models for young objects. The surface gravity results for the later-type young L dwarfs are all higher than predicted by the evolutionary models, but not unphysical for field objects, and the $H$-band fits are closest to the predictions from evolutionary models (Fig. 12), where the spectral shape is expected to be particularly sensitive to surface gravity because of decreased collisionally induced absorption from $\mathrm{H}_{2}$ relative to $\mathrm{H}_{2} \mathrm{O}$ opacity (see, e.g., Rice et al. [2011], Fig. 6).

Inconsistencies between model fits from individual bands, evident in both the probability distributions and in the bestfit spectra, likely stem from the dust treatment in the dusty models, which do not incorporate enhanced dust content, particularly in small grains, expected for low-surface-gravity atmospheres (e.g., Schmidt et al. 2008; Marocco et al. 2014). These results support the growing consensus that additional opacity sources and more sophisticated dust and cloud treatments (e.g., Witte et al. 2009; Barman et al. 2011a; Morley et al. 2012, 2014; Allard 2014) are necessary for modeling the atmospheres of young brown dwarfs as well as of directly imaged exoplanets.
These young, low-mass objects in particular illustrate the necessity of comparing observations in multiple bands in order to test the reliability of spectral characterization. Thus, we caution against using results of model fits to low-resolution spectra to confirm or rule out youth of very low-mass objects. Similar results for the P1640 spectrum of $\kappa$ Andromedae B are described in Hinkley et al. (2013).

\subsection{Signal-to-Noise Ratio}

The probability distributions, best-fit parameters, and their uncertainties described above were calculated using the $\geq 10 \%$ uncertainties assigned to the simulated spectra described in $\S 2.2$. The noise level was selected such that the highest flux point of each simulated spectrum had $\mathrm{S} / \mathrm{N}=10$, which is expected to be typical for spectra of exoplanets from high-contrast integral field spectrographs (e.g., Oppenheimer et al. 2013; Chilcote et al. 2015). The actual uncertainty on the binned flux points (i.e., the uncertainty on the observed flux points within the spectral range of each P1640 wavelength channel, added in quadrature) are significantly lower than $10 \%$ because the original SpeX and GNIRS template spectra have significantly higher $\mathrm{S} / \mathrm{N}$ and we have not adjusted the binned flux values at all. This begs the question of whether the results described above are truly representative of model fits to $\mathrm{S} / \mathrm{N} \leq 10$ spectra.

Therefore, we use two Monte Carlo simulations to test the dependence of the best-fit parameters and their uncertainties on the adopted S/N of the simulated spectrum. The first Monte Carlo test was applied to all spectral types with the adopted $(\mathrm{S} / \mathrm{N} \leq 10)$ uncertainties, and the second test used the M8, L5, and T4.5 simulated spectra to compare results from a range of different adopted uncertainty values. The complete $Y J H$ simulated spectra were used for both $\mathrm{S} / \mathrm{N}$ tests, and both tests compare simulated spectra to the calculated model spectral grid (i.e., $50 \mathrm{~K}$ intervals in effective temperature and $0.1 \mathrm{dex}$ intervals in surface gravity; Fig. 3), without the interpolation used in the MCMC fitting.

\subsubsection{Resampling versus Real $S / N$}

The first $\mathrm{S} / \mathrm{N}$ test, performed for all spectral types, resamples the simulated spectra by adding a random number to the binned flux values from the template spectra. The random number is drawn from a Gaussian distribution the width of the adopted noise level $(\mathrm{S} / \mathrm{N} \leq 10)$, hereafter called a "resampled" simulated spectrum. For $10^{4}$ resampled simulated spectra, we calculate the goodness-of-fit to each calculated model spectrum, define the minimum goodness-of-fit value as the best-fit parameters, and calculate the mean and standard deviation of the $10^{4}$ best-fit values.

The mean best-fit parameters for $10^{4}$ resampled spectra of each template object are consistent with the $Y J H$ results listed in Table 2 to within one standard deviation. The largest discrepancies are for the M5 field object in surface gravity (different by 
$\sim 0.5$ dex) and the $\mathrm{L} 0 \gamma$ object in effective temperature (different by $\sim 60 \mathrm{~K}$ ), both still within $1-\sigma$ uncertainties of the MCMC results. Interestingly, the $\mathrm{L} 0 \gamma$ object also has the worst "best-fit" $Y J H$ spectrum (see Fig. 10). The uncertainties were generally about the same or smaller (by up to a factor of 2) for the resampled spectrum fits, but this is possibly a consequence of using only the model grid instead of allowing for linear interpolation between calculated models.

\subsubsection{Dependence of Uncertainty on $S / N$}

For the second S/N test, we use the simulated spectra for the M5, L1, and T4.5 field objects to test the dependence of best-fit parameters on $\mathrm{S} / \mathrm{N}$. As with the first test, the flux points are all resampled from within a Gaussian distribution the width of the noise on that flux point, this time for 20 values of maximum $\mathrm{S} / \mathrm{N}$, ranging from $\mathrm{S} / \mathrm{N}=2$ to 100 . Each template spectrum is resampled $10^{4}$ times for each $\mathrm{S} / \mathrm{N}$ value, and the best-fit model has the minimum goodness-of-fit value when compared to the resampled spectrum. The mean and standard deviation of the best-fit parameters from $10^{4}$ trials are plotted as a function of $\mathrm{S} / \mathrm{N}$ in Figure 15.

The best-fit parameters for the resampled simulated spectra approach $T_{\text {eff }}=2950 \mathrm{~K}$ and $\log (g)=5.4$ for the M5, $T_{\text {eff }}=$ $2300 \mathrm{~K}$ and $\log (g)=5.5$ for the $\mathrm{L} 1$, and $T_{\text {eff }}=1200 \mathrm{~K}$ and $\log (g)=5.3$ for the T4.5. These values are consistent with the MCMC YJH results within the $1-\sigma$ uncertainties, but some are offset from the closet model grid point to the MCMC result, likely because of the fundamental difference between MCMC and purely Monte Carlo techniques and the use of interpolation versus the calculated model grid.

By $\mathrm{S} / \mathrm{N}>5$, the mean temperatures for all three objects converge to within a few percent of the $\mathrm{S} / \mathrm{N}=100$ value, and the surface gravities converges to the $\mathrm{S} / \mathrm{N}=100$ values for $\mathrm{S} / \mathrm{N}>10$. The uncertainties in surface gravity remain large ( $\sim .15$ dex) for the M5 dwarf, likely because the low-resolution near-infrared spectra of $\mathrm{M}$ dwarfs are not as sensitive to gravity as the spectra of cooler objects are (i.e., Fig. 5).

It should be noted that these tests assume a Gaussian noise model for the simulated IFS spectra, which may not be the most appropriate noise model for low S/N data. However, the Gaussian model is reasonable for a long chain of error sources with different characteristics, as is the case for high-contrast IFS data. We expect the effects of the assumed error distribution to be smaller than the effect of the instrumental and the model systematics; therefore, we leave the evaluation of different noise models for a future paper. We also ignore instrumental systematic uncertainties mentioned in $\S \S 2.2$ and 5.3. However, Oppenheimer et al. (2013) tested the fidelity of their S4 spectral extraction method by injecting fake sources with a T4.5 spectrum into the data cubes obtained for HR 8799bcde and comparing the extracted spectra to the input spectrum. The average deviation from the input spectrum over all wavelengths was $\sim 2-9 \%$, except for the innermost planet HR $8799 \mathrm{e}$, which

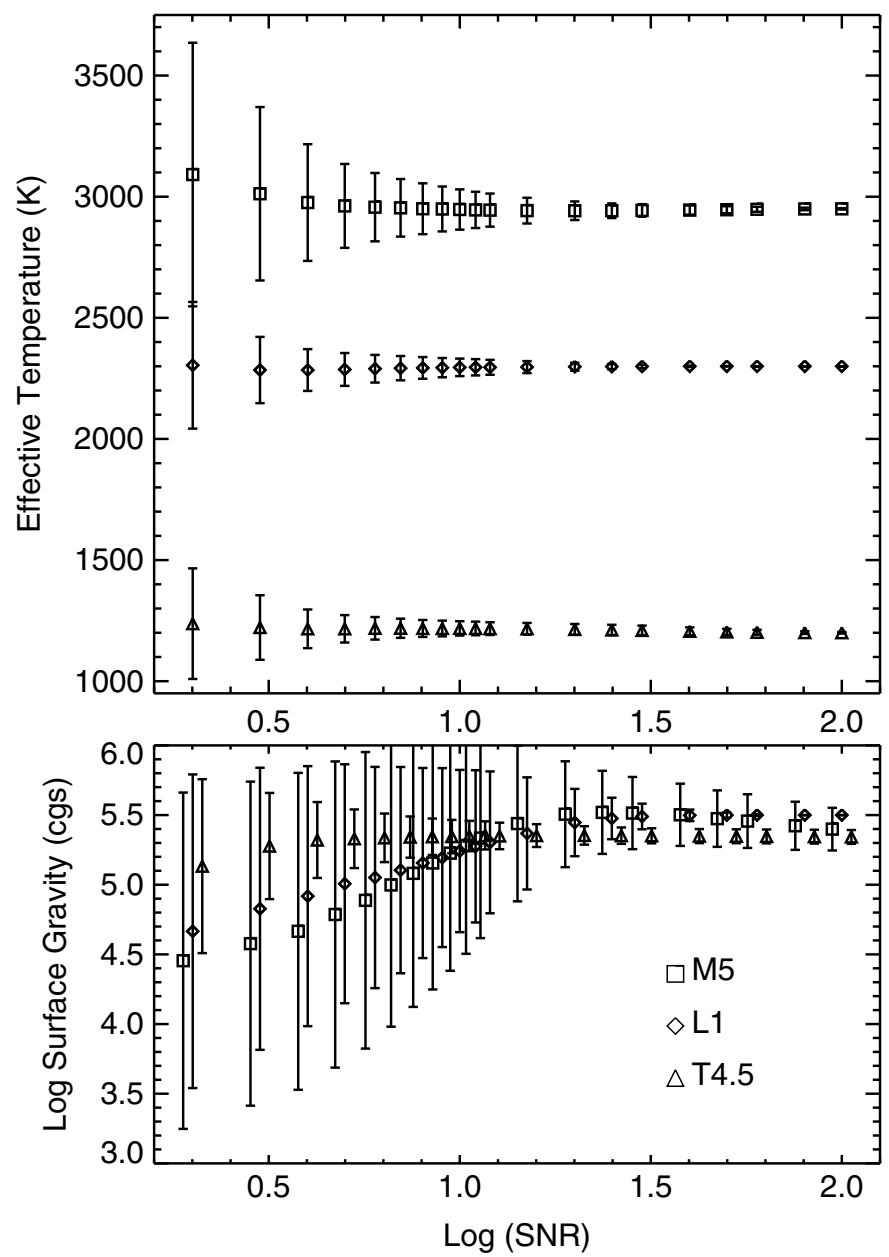

FIG. 15.-Best-fit effective temperatures (top) and surface gravities (bottom) for fits to the simulated P1640 spectra of the M5 (squares), L1 (diamonds), and $\mathrm{T} 4.5$ (triangles) dwarf templates with varied $\mathrm{S} / \mathrm{N}$, as described in $\S 5.7$. Symbols on the bottom panel are offset slightly in $\log (\mathrm{S} / \mathrm{N})$ for clarity. The error bars show the standard deviation of the distribution of best-fit parameters for $10^{4}$ resampled spectra using comparisons with the noninterpolated model grid. The results suggest that $\mathrm{S} / \mathrm{N} \geq 5$ is required for temperature determination, and $\mathrm{S} / \mathrm{N} \sim 10$ is optimal for inferring surface gravity from best-fit model spectra.

was $15 \%$. Therefore, we expect the instrumental systematics to be below the $\mathrm{S} / \mathrm{N} \sim 10$ level we adopt for the simulated spectra. It should also be noted that the errors bars on Figure 15 are not representative of the actual uncertainty in the best-fit parameters at high $\mathrm{S} / \mathrm{N}$, just the distribution of best-fit parameters for resampled spectra, which will always approach a delta function at high $\mathrm{S} / \mathrm{N}$ even for poor quality fits.

\section{CONCLUSIONS}

We have developed a robust spectral fitting method and tested it for low-resolution ( $R \sim 30$ to 60$)$ near-infrared $(Y J H)$ simulated spectra created from higher resolution observed template spectra of MLT dwarfs. We used the fitting method to explore the sensitivity of these spectra to effective 
temperature and surface gravity using the limiting case dust treatments of the dusty and cond PHOENIX atmosphere models for $\mathrm{M} / \mathrm{L}$ dwarfs and $\mathrm{T}$ dwarfs, respectively.

The PHOENIX dusty and cond models reproduce the simulated spectra of both field and young objects when the entire wavelength regime is used in the fitting procedure, despite the limiting case dust treatments in these models. Best-fit spectra determined using only one band ( $Y J$ or $H$ ) are typically within the adopted uncertainties of the complete simulated spectra for the field dwarfs, with some exceptions for the L5, L8, and $\mathrm{T}$ dwarfs. However, the single-band best-fits generally do not reproduce the spectrum in the other band for spectra of young objects, which leads us to recommend caution when using single bands of low-resolution spectra to characterize young, lowmass objects.

Our results indicate that low-resolution near-infrared spectra are sensitive to temperature with precisions as good as $\pm 50-100 \mathrm{~K}$ for $\mathrm{L}$ and $\mathrm{T}$ dwarfs, while the constraints are much looser ( $\pm 100-500)$ for $\mathrm{M}$ dwarfs. The low-resolution nearinfrared spectra are increasingly sensitive to surface gravity at later spectral types, although with lower precision (up to $\sim 1$ dex for hotter objects) and significant offsets from predictions of evolutionary models for the young objects.

The complete and trimmed $Y J H$ spectra produced similar results and uncertainties, indicating that losing a few flux points to poor sky subtraction, speckle suppression, or other reduction and extraction effects will not significantly affect the characterization of the companion.

The best-fit parameters and uncertainties can be considerably different for the $Y J$ and $H$ subsets of the complete $Y J H$ spectrum, especially for temperature. Surface gravity results are generally consistent within the (large) uncertainties, but are discrepant from predictions of evolutionary models (see below). These results suggest that high-contrast IFS observing campaigns should characterize detected companions with as broad a wavelength coverage as possible. In future analysis we will expand our simulated spectra into the $K$-band and test the sensitivity and accuracy of different filter combinations to model parameters.

The temperatures from the $Y J$ spectral fits are typically more consistent with the temperatures from the full $Y J H$ fits and closer to temperatures derived for objects of the same spectral types using other methods, with the notable exceptions of the young L5 $\gamma$ and L7 VL-G objects, for which the $H$-band fits are closer to complete $Y J H$ results (Fig. 11).

The best-fit temperatures are most discrepant from literature values for the field L5 and L8 objects, likely the result of our use of the PHOENIX dusty models. The best-fit gravities are generally consistent with evolutionary models for the field objects, but are significantly discrepant for the young objects. The complete $Y J H$ spectra fits to young objects always produced high surface gravities. Even $H$-band spectra, which have been shown to be gravity-sensitive at higher spectral resolutions (Lucas et al. 2001; Luhman et al. 2005; Allers et al. 2007; Rice et al. 2010c, 2011), only produced marginally consistent results for the young L dwarfs (see Fig. 12). Thus we caution against using model fits to low-resolution nearinfrared spectra to confirm or rule out youth of very low-mass companions.

We advise that even good fits should be treated with caution when interpreting fits to low-resolution near-infrared spectra for very low-mass objects. The results for simulated spectra from $\mathrm{L}-\mathrm{T}$ transition objects and for young objects in particular highlight the need for testing the more sophisticated dust treatments now available in cool atmosphere models (e.g., Morley et al. 2012) and for comparing best-fit parameters over a broad wavelength range.

We determine that a minimum of $\mathrm{S} / \mathrm{N} \sim 5$ is required to reliably constrain the temperature of a low-mass companion and that $\mathrm{S} / \mathrm{N} \geq 10$ is ideal for constraining surface gravity. We caution that these results are based on tests using objects with spectra that are reasonably well reproduced by the PHOENIX dusty and cond models and may not be representative of results for all directly imaged high-contrast companions.

Future work will expand this analysis to simulated spectra for GPI and SPHERE, which cover $Y J H K$ with several individual filters, and to include varying metallicity and dust treatments in the atmosphere models we consider.

The authors thank K. Cruz, J. Faherty, the BDNYC research group, \#AstroHackNY, and T. Barman, D. Brenner, I. Crossfield, S. Douglas, D. Foreman-Mackey, A. Kraus, L. Hillenbrand, N. Madhusudhan, and A. Price-Whelan for useful discussions. We also thank the anonymous referee for both broad and detailed comments that helped us improve the manuscript.

This research was supported in part by the American Astronomical Society's Small Research Grant Program, NASA Astrophysics Data Analysis Program (ADAP) award 11ADAP11-0169, and by the National Science Foundation under Grant No. 1211568. A portion of this work was supported by NASA Origins of the Solar System Grant No. NMO7100830/ 102190. A portion of the research in this paper was carried out at the Jet Propulsion Laboratory, California Institute of Technology, under a contract with the National Aeronautics and Space Administration (NASA) and was funded by internal Research and Technology Development funds. In addition, part of this work was performed under a contract with the California Institute of Technology (Caltech) funded by NASA through the Sagan Fellowship Program. The members of the Project 1640 team are also grateful for support from the Cordelia Corporation, Hilary and Ethel Lipsitz, the Vincent Astor Fund, Judy Vale, Andrew Goodwin, and an anonymous donor. This research has made use of the IRTF Spectral Library, the SIMBAD database, operated at CDS, Strasbourg, France, and NASA's Astrophysics Data System. 


\section{REFERENCES}

Allard, F. 2014, in IAU Symp. 299, Exploring the Formation and Evolution of Planetary Systems, eds. M. Booth, B. C. Matthews, \& J. R. Graham (Cambridge: Cambridge Univ. Press), 271-272

Allers, K. N., Jaffe, D. T., Luhman, K. L., Liu, M. C., Wilson, J. C., Skrutskie, M. F., Nelson, M., Peterson, D. E., et al. 2007, ApJ, 657,511

Allers, K. N. \& Liu, M. C. 2013, ApJ, 772, 79

Baraffe, I., Chabrier, G., Allard, F., \& Hauschildt, P. H. 2002, A\&A, 382,563

Baraffe, I., Chabrier, G., Barman, T. S., Allard, F., \& Hauschildt, P. H. 2003, A\&A, 402, 701

Barman, T. S., Macintosh, B., Konopacky, Q. M., \& Marois, C. 2011a, ApJ, 733, 65

. 2011b, ApJ, 735, L 39

Beichman, C. A., Krist, J., Trauger, J. T., Greene, T., Oppenheimer, B., Sivaramakrishnan, A., Doyon, R., Boccaletti, A., et al. 2010, PASP, 122,162

Beuzit, J., Feldt, M., Dohlen, K., Mouillet, D., Puget, P., Wildi, F., Abe, L., Antichi, J., et al. 2008, in Proc. SPIE, 7014, 12 pp

Bowler, B. P., Liu, M. C., Dupuy, T. J., \& Cushing, M. C. 2010, ApJ, 723, 850

Bowler, B. P., Liu, M. C., Shkolnik, E. L., \& Dupuy, T. J. 2013, ApJ, 774, 55

Burrows, A., Marley, M., Hubbard, W. B., Lunine, J. I., Guillot, T., Saumon, D., Freedman, R., Sudarsky, D., et al. 1997, ApJ, 491, 856

Chabrier, G., Baraffe, I., Allard, F., \& Hauschildt, P. 2000, ApJ, 542,464

Chilcote, J., Barman, T., Fitzgerald, M. P., Graham, J. R., Larkin, J. E., Macintosh, B., Bauman, B., Burrows, A. S., et al. 2015, ApJ, 798, L 3

Chun, M., Toomey, D., Wahhaj, Z., Biller, B., Artigau, E., Hayward, T., Liu, M., Close, L., et al. 2008, Proc. SPIE, 7015, 9 pp

Crepp, J. R., Rice, E. L., Veicht, A., Aguilar, J., Pueyo, L., Giorla, P., Nilsson, R., Luszcz-Cook, S. H., et al. 2015, ApJ, 798, L 43

Cruz, K. L., Kirkpatrick, J. D., \& Burgasser, A. J. 2009, AJ, 137, 3345

Currie, T., Burrows, A., Girard, J. H., Cloutier, R., Fukagawa, M., Sorahana, S., Kuchner, M., Kenyon, S. J., et al. 2014, ApJ, 795, 133

Cushing, M. C., Marley, M. S., Saumon, D., Kelly, B. C., Vacca, W. D., Rayner, J. T., Freedman, R. S., Lodders, K., et al. 2008, ApJ, 678, 1372

Cushing, M. C., Rayner, J. T., \& Vacca, W. D. 2005, ApJ, 623, 1115

del Burgo, C., Martín, E. L., Zapatero Osorio, M. R., \& Hauschildt, P. H. 2009, A\&A, 501, 1059

Deming, D., Harrington, J., Laughlin, G., Seager, S., Navarro, S. B., Bowman, W. C., \& Horning, K. 2007, ApJ, 667, L 199

Elias, J. H., Joyce, R. R., Liang, M., Muller, G. P., Hileman, E. A., \& George, J. R. 2006, Proc. SPIE, 6269

Faherty, J. K., Rice, E. L., Cruz, K. L., Mamajek, E. E., \& Núñez, A. 2013, AJ, 145, 2

Fergus, R., Hogg, D. W., Oppenheimer, R., Brenner, D., \& Pueyo, L. 2014, ApJ, 794, 161

Fortney, J. J., Marley, M. S., \& Barnes, J. W. 2007, ApJ, 659, 1661

Fortney, J. J., Marley, M. S., Saumon, D., \& Lodders, K. 2008, ApJ, 683,1104

Fortney, J. J., Mordasini, C., Nettelmann, N., Kempton, E. M.-R., Greene, T. P., \& Zahnle, K. 2013, ApJ, 775, 80
Gagné, J., Lafrenière, D., Doyon, R., Malo, L., \& Artigau, É. 2014, ApJ, 783, 121

Hauschildt, P. H., Allard, F., \& Baron, E. 1999, ApJ, 512, 377

Hauschildt, P. H., Baron, E., \& Allard, F. 1997, ApJ, 483, 390

Hinkley, S., Monnier, J. D., Oppenheimer, B. R., Roberts, L. C., Jr., Ireland, M., Zimmerman, N., Brenner, D., Parry, I. R., et al. 2011a, ApJ, 726, 104

Hinkley, S., Oppenheimer, B. R., Brenner, D., Parry, I. R., Sivaramakrishnan, A., Soummer, R., \& King, D. 2008, Proc. SPIE, 7015, $10 \mathrm{pp}$

Hinkley, S., Oppenheimer, B. R., Brenner, D., Zimmerman, N., Roberts, L. C., Parry, I. R., Soummer, R., Sivaramakrishnan, A., et al. 2010, ApJ, 712, 421

Hinkley, S., Oppenheimer, B. R., Zimmerman, N., Brenner, D., Parry, I. R., Crepp, J. R., Vasisht, G., Ligon, E., et al. 2011b, PASP, 123, 74

Hinkley, S., Pueyo, L., Faherty, J. K., Oppenheimer, B. R., Mamajek, E. E., Kraus, A. L., et al. 2013, ApJ, 779, 153

Janson, M., Bergfors, C., Goto, M., Brandner, W., \& Lafrenière, D. 2010, ApJ, 710, L 35

Kalas, P., Graham, J. R., Chiang, E., Fitzgerald, M. P., Clampin, M., Kite, E. S., Stapelfeldt, K., Marois, C., et al. 2008, Science, 322,1345

Kirkpatrick, J. D., Barman, T. S., Burgasser, A. J., McGovern, M. R., McLean, I. S., Tinney, C. G., \& Lowrance, P. J. 2006, ApJ, 639, 1120

Knutson, H. A., Charbonneau, D., Allen, L. E., Burrows, A., \& Megeath, S. T. 2008, ApJ, 673, 526

Konopacky, Q. M., Barman, T. S., Macintosh, B. A., \& Marois, C. 2013, Science, 339, 1398

Lagrange, A., Bonnefoy, M., Chauvin, G., Apai, D., Ehrenreich, D., Boccaletti, A., Gratadour, D., Rouan, D., et al. 2010, Science, 329, 57

Liu, M. C., Magnier, E. A., Deacon, N. R., Allers, K. N., Dupuy, T. J., Kotson, M. C., Aller, K. M., Burgett, W. S., et al. 2013, ApJ, 777, L 20

Lucas, P. W., Roche, P. F., Allard, F., \& Hauschildt, P. H. 2001, MNRAS, 326, 695

Luhman, K. L., Lada, E. A., Muench, A. A., \& Elston, R. J. 2005, ApJ, 618,810

Luhman, K. L., Patten, B. M., Marengo, M., Schuster, M. T., Hora, J. L., Ellis, R. G., Stauffer, J. R., Sonnett, S. M., et al. 2007, ApJ, 654, 570

Luhman, K. L. \& Rieke, G. H. 1999, ApJ, 525, 440

Macintosh, B., Graham, J. R., Ingraham, P., Konopacky, Q., Marois, C., Perrin, M., Poyneer, L., Bauman, B., et al. 2014, PNAS, 111,12661

Macintosh, B. A., Graham, J. R., Palmer, D. W., Doyon, R., Dunn, J., Gavel, D. T., Larkin, J., Oppenheimer, B., et al. 2008, Proc. SPIE, 7015, 13 pp

Marocco, F., Day-Jones, A. C., Lucas, P. W., Jones, H. R. A., Smart, R. L., Zhang, Z. H., Gomes, J. I., Burningham, B., et al. 2014, MNRAS, 439, 372

Marois, C., Macintosh, B., Barman, T., Zuckerman, B., Song, I., Patience, J., Lafrenière, D., \& Doyon, R. 2008, Science, 322, 1348

Marois, C., Zuckerman, B., Konopacky, Q. M., Macintosh, B., \& Barman, T. 2010, Nature, 468, 1080 
McElwain, M. W., Brandt, T. D., Janson, M., Knapp, G. R., Peters, M. A., Burrows, A. S., Carlotti, A., Carr, M. A., et al. 2012, Proc. SPIE, 8446, $10 \mathrm{pp}$

Metchev, S. A. \& Hillenbrand, L. A. 2006, ApJ, 651, 1166

Mohanty, S., Stassun, K. G., \& Doppmann, G. W. 2010, ApJ, 722,1138

Morley, C. V., Fortney, J. J., Marley, M. S., Visscher, C., Saumon, D., \& Leggett, S. K. 2012, ApJ, 756, 172

Morley, C. V., Marley, M. S., Fortney, J. J., Lupu, R., Saumon, D., Greene, T., \& Lodders, K. 2014, ApJ, 787, 78

Öberg, K. I., Murray-Clay, R., \& Bergin, E. A. 2011, ApJ, 743, L 16 Oppenheimer, B. R., Baranec, C., Beichman, C., Brenner, D., Burruss, R., Cady, E., Crepp, J. R., Dekany, R., et al. 2013, ApJ, 768, 24

Oppenheimer, B. R., Beichman, C., Brenner, D., Burruss, R., Cady, E., Crepp, J., Hillenbrand, L., Hinkley, S., et al. 2012, Proc. SPIE, 8447, 13 pp

Oppenheimer, B. R. \& Hinkley, S. 2009, ARA\&A, 47, 253

Patience, J., King, R. R., de Rosa, R. J., \& Marois, C. 2010, A\&A, 517, A $76+$

Patience, J., King, R. R., De Rosa, R. J., Vigan, A., Witte, S., Rice, E., Helling, C., \& Hauschildt, P. 2012, A\&A, 540, A 85

Pueyo, L., Crepp, J. R., Vasisht, G., Brenner, D., Oppenheimer, B. R., Zimmerman, N., Hinkley, S., Parry, I., et al. 2012, ApJS, 199, 6

Rayner, J. T., Cushing, M. C., \& Vacca, W. D. 2009, ApJS, 185, 289

Rayner, J. T., Toomey, D. W., Onaka, P. M., Denault, A. J., Stahlberger, W. E., Vacca, W. D., Cushing, M. C., \& Wang, S. 2003, PASP, 115,362

Rice, E. L., Barman, T., Mclean, I. S., Prato, L., \& Kirkpatrick, J. D. 2010a, ApJS, 186, 63

Rice, E. L., Barman, T. S., McLean, I. S., \& Kirkpatrick, J. D. 2010b, BAAS, 42, 335
Rice, E. L., Faherty, J. K., Cruz, K., Barman, T., Looper, D., Malo, L., Mamajek, E. E., Metchev, S., et al. 2011, in ASP Conf. Ser. 448, 16th Cambridge Workshop on Cool Stars, Stellar Systems, and the Sun, eds. C. Johns-Krull, M. K. Browning, \& A. A. West (San Francisco: ASP), 481

Rice, E. L., Faherty, J. K., \& Cruz, K. L. 2010c, ApJ, 715, L 165

Roberts, L. C., Jr., Rice, E. L., Beichman, C. A., Brenner, D., Burruss, R., Crepp, J. R., Dekany, R. G., Hillenbrand, L. A., et al. 2012, AJ, 144, 14

Rojas-Ayala, B., Covey, K. R., Muirhead, P. S., \& Lloyd, J. P. 2012, ApJ, 748, 93

Schlieder, J. E., Lépine, S., Rice, E., Simon, M., Fielding, D., \& Tomasino, R. 2012, AJ, 143, 114

Schmidt, T. O. B., Neuhäuser, R., Seifahrt, A., Vogt, N., Bedalov, A., Helling, C., Witte, S., \& Hauschildt, P. H. 2008, A\&A, 491, 311

Siess, L., Dufour, E., \& Forestini, M. 2000, A\&A, 358, 593

Sing, D. K. \& López-Morales, M. 2009, A\&A, 493, L 31

Sparks, W. B. \& Ford, H. C. 2002, ApJ, 578, 543

Spiegel, D. S. \& Burrows, A. 2012, ApJ, 745, 174

Stephens, D. C., Leggett, S. K., Cushing, M. C., Marley, M. S., Saumon, D., Geballe, T. R., Golimowski, D. A., Fan, X., et al. 2009, ApJ, 702, 154

Suzuki, R., Kudo, T., Hashimoto, J., Carson, J., Egner, S., Goto, M., Hattori, M., Hayano, Y., et al. 2010, Proc. SPIE, 7735

Witte, S., Helling, C., \& Hauschildt, P. H. 2009, A\&A, 506, 1367

Zimmerman, N., Brenner, D., Oppenheimer, B. R., Parry, I. R., Hinkley, S., Hunt, S., \& Roberts, R. 2011, PASP, 123, 746

Zimmerman, N., Oppenheimer, B. R., Hinkley, S., Brenner, D., Parry, I. R., Sivaramakrishnan, A., Hillenbrand, L., Beichman, C., et al. 2010, ApJ, 709, 733 